\title{
《The principle of natural law》 show "Complete Scientific Modeling" with its internet could inclusive of all human faith, knowledge and experience. The world peace come from all the personality together blooming and complement each other
}

\author{
Yansha Yu-Sandstrom \\ Chinese American. Independent scholar \\ Address: Hannam dong Itaewan gu, Seoul 140-210 \\ yusands@gmail.com
}

\begin{abstract}
This paper, based on 《Humankind's interdisciplinary and complete theory of mutual cognition》 publishing by china "Xian Zhuang Publisher", and 《The principle of cosmic life being multi-dimensional and simultaneously evolving》Published by "Frontier Science" 2014 Vol. Issue 2 China, Abbreviation 《The principle of natural law》.

For example: The complete universe is a solid ball. All human's cognitions and certifications are this solid ball itself and each of its levels, angles, positions, interfaces... "Presence Ways and Presence Relationships". When all human's cognitions and certifications, departure from their respective standpoint and attributes, reaches to each other's standpoint and attributes. That will become same one song played in different tones (as each other or each own). Otherwise people will each stick to their own limited area, disagree and no-inclusive other side.

Basis on the above, 《The principle of natural law》don't against any of human experience and knowledge. It clarifies all human cognitions and certifications where are they located at the system of universe, what people has been find out or not grasp yet. How could from people knowing arrival to their unknowing, and final build all human consensus.

For help different disciplines reach consensus, 《The principle of natural law》 will combine with each disciplines examples, and follow three big basic theorem steps to show that same one song how could play with different tones.

\section{Indexing terms/Keywords}

The laws of nature. Interdisciplinary. Intercross and Integration. Cross-Integration. overlapping and conformity. Complete Mathematical modeling. Theoretical Physics. Universal Unified Field. Einstein's theory of relativity. Cosmological Constant. Wave-particle duality. Particle spin. Trinity. I Ching. The Book of Changes. Tao Te Ching. Bible. The principle of Bible. Quran. The principle of Quran. Multidimensional. Synchronous Evolution. Darwin's theory of evolution.
\end{abstract}

\section{Academic Discipline And Sub-Disciplines}

Mathematics-Computers-Physics-Chemistry-Philosophy-Religion.

\section{SUBJECT CLASSIFICATION}

Mathematics-Computers-Physics-Chemistry-Philosophy-Religion, Graph, Symbolism,

\section{TYPE (METHOD/APPROACH)}

《The principle of natural law》 have three big basic theorem steps inclusive to show and unfold mankind all the cognition certification.

\section{Council for Innovative Research}

Peer Review Research Publishing System

Journal: JOURNAL OF ADVANCES IN MATHEMATICS

Vol .11, No.8

www.cirjam.com, editorjam@gmail.com 
Universal Unified Field System of Science

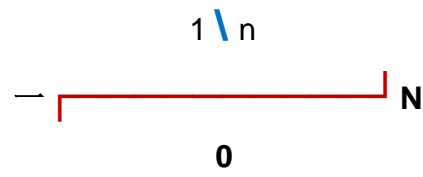

Above the same one song played in different tone as below

Particle Perfect Spin System of Science

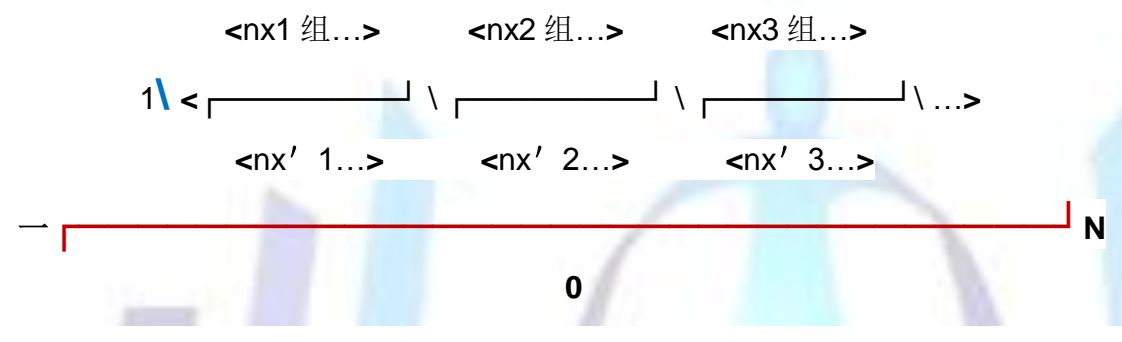

Above the same one song played in different tone as below

Positive and Negative Numbers Perfeck System of Science

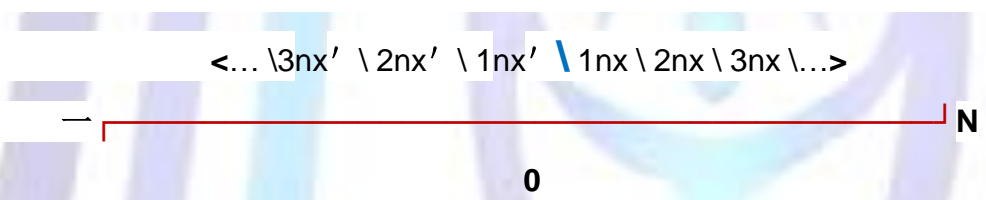

Above the same one song played in different tone as below

TAI CHI Systerm of The Book of Changes

YONG YI

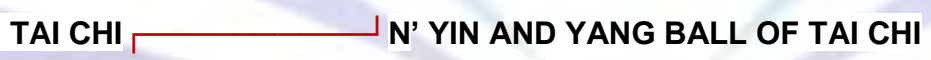

YIN YI

Above the same one song played in different tone as below

"Tao System" of "Tao Te Ching"

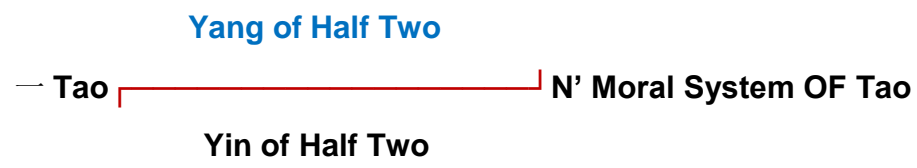

Above the same one song played in different tone as below

Trinity of Bible 
HOLY BODY

一 God $\longrightarrow$ N' TRINITY OF GOD

HOLY GHOST

Above the same one song played in different tone as below

Three Kayas Buddha of Tantric

MALE BODY BUDDHA

\section{BUDDHA \\ N' THREE KAYAS BUDDHA \\ FEMALE BODY BUDDHA}

Etc.

Theorem one: "Cornerstone of principle--Six composite concepts of Quadrity" and "Complete Universe's own department of Trinity".

\section{Theorem Two: Include 《Natural Human Development Brief History》:}

From synchronous multi-D mutual-birth generate frequency, synchronous multi-D mutual-rebirth generate frequency cycle, Principle show and unfold in six composite concepts Quadrity, sync compound (immortal, immortal with have-life-havedeath, have-life-have-death)-renew division and integration mutual-rebirth evolve each own and mutual presence ways and presence relationships and build in Complete mathematical modeling.

\section{Theorem Three:}

"Basis's own department with its naturally composite correspond standpoint and attributes" and "Persons-Concepts、 Position、Trend, these three key elements, jointly determine Six composite concepts of Quadrity, whole corresponding, consubstantiality relative, at same time sync keep (and or) conversion respective self or each other, naturally composite correspond standpoint and attributes"-- Complete Relativistic and Complete Dialectics.

Summary

\section{《The principle of natural law》}

This paper, based on 《Humankind's interdisciplinary and complete theory of mutual cognition》 publishing by china "Xian Zhuang Publisher", and 《The principle of cosmic life being multi-dimensional and simultaneously evolving》Published by "Frontier Science" 2014 Vol. Issue 2 China, Abbreviation《The principle of natural law》.

For example: The complete universe is a solid ball. All human's cognitions and certifications are this solid ball itself and each of its levels, angles, positions, interfaces... "Presence Ways and Presence Relationships". When all human's cognitions and certifications, departure from their respective standpoint and attributes, reaches to each other's standpoint and attributes. That will become same one song played in different tones (as each other or each own). Otherwise people will each stick to their own limited area, disagree and no-inclusive other side.

Basis on the above, 《The principle of natural law》don't against any of human experience and knowledge. It clarifies all human cognitions and certifications where are they located at the system of universe, what people has been find out or not grasp yet. How could from people knowing arrival to their unknowing, and final build all human consensus.

For help different disciplines reach consensus, 《The principle of natural law》 will combine with each disciplines examples, and follow three big basic theorem steps to show that same one song how could play with different tones.

This article based on sound of "natural numbers, set of natural numbers" of mathematical foundations of science. Mathematical field chaos application and often confuse the conservation law, the cosmological constant of Mathematical field carding clear as: 1) Proportion that immortal equal amount renewed and natural no change number, natural change number sets". 2) Proportion have life have death to probability about equal amount renew and natural number often change, natural number sets always change. Thereby by a sound scientific unified modeling, building an inclusive Internet complete system procedures, for earth humanity reach the reconciliation and consensus, together save life from multidimensional complex survival crisis, open up a shortcut.

Including scientific, because many disciplines ideas not fully completed, the paper like this will often gave examples of different disciplines of experiences and knowledge, complementary clarify inclusive complete new names and the complete new methods, run neck and neck with ancient wisdom.

Theorem one: "Cornerstone of principle--Six composite concepts of Quadrity" and "Complete Universe's own department of Trinity" 
All great civilization and culture around the world tell us, once people mastery their own and others experiences and knowledge, human all the cognitions and certifications will naturally follow "Complete Universe System" its own dual trend (e.g. vertical axis) of division and integration, "Commonness principles" at same time is "personalized law" summarize-generalize into "one to six composite concepts" of "one to four persons of Quadrity " (e.g. horizontal axis), understand "Universe's own department of Completely Trinity"; At same time, vice versa. Problems of the universe and life, start from here clarification.

First of all, 《The principle of natural law》synchronization human's transition development of experience and knowledge, connect with the foregoing and carry forward to a new paragraph, according to "synchronous multi-D birth self and each other that generate frequency, Synchronous multi-D rebirth self and each other that generate frequency cycle"; Universe follow the frequency, permutations combination everything position. Universe are science ultimate structure "Frequency type permutation combination order", "Zero frequency type non permutation combination orderly link" with "Frequency type permutation combination orderly link", "Frequency type permutation combination orderly system" show and expand. Among them, Physics "Wave-particle duality", that "waves" come from "Frequency", that "particle" come from "permutation combination order". Reference Bible had "One substance in three persons of Trinity", and "person" is "Presence Ways of Position", specific and detailed build that consistent with the scientific characteristics of the times "One substance in four persons of Six composite concepts of Quadrity" as cornerstone of principle:

\section{Section 1. From human function formatting "Complete Universe"}

\section{First person one composite concepts -}

"-〔N (1-link\n-links、0-link) ] of triple sync completely compound immortal-renew division and integration mutualrebirth evolve frequency type permutation-combination orderly spin system".

Abbreviation:

"-〔N (1-link\n-links、0-link) 〕 of triple completely compound spin system".

Or, "- $[N(1 \backslash n, 0)] "$.

Or, "一".

Or, "N".

Etc.

【Above same one song played in different tone as Mathematics:

“- $\{\mathrm{N}[1 / 2$ proportion immortal equal amount renew and natural number sets change mathematical formula $〔(1-$ naturalnumber with its amount have life have death change) $\backslash$ (n-number-set with its amount have life have death change) ] 、1/2 proportion immortal equal amount renew and 0 natural number never change] of triple sync completely compound immortal-renew division and integration mutual-rebirth evolve proportion equal amount renew frequency type permutationcombination orderly natural number-set mutative spin system\}".

Abbreviation: " $-[N(1 \backslash n, 0)]$ of triple sync completely compound immortal-renew division and integration mutualrebirth evolve proportion equal amount renew frequency type permutation-combination orderly natural number-set mutative spin system"; Or, " $-\lceil N(1 \backslash n, 0) 〕$ of triple proportion immortal equal amount renew and natural number sets change spin system"; Or same as above.

"First person one composite concepts - " its "- of triple" composite correspond "Second person two composite concepts 二.A、二.B" each "one coincide" establish; at same time vice versa. Known one or two of them, also need to know other one or two of them; otherwise human certification cognition always unable to have understand and balance syncretic fully inclusive.

\{ 'Second person one of two composite concepts 二.A" " $1 / 2$ proportion immortal equal amount renew and natural number sets change mathematical formula 〔(1-natural-number with its amount life and death change) $\backslash$ (n-number-set with its amount life and death change) ] "\}, and, ("Second person one of two composite concepts 二.B" " $1 / 2$ proportion immortal equal amount renew and 0 natural number never change"), at exactly same one position, whole corresponding, consubstantiality relative, sync compound immortal-renew division and integration mutual-rebirth equal amount exchange evolve each other at same time, at exactly same one position, sync compound immortal-renew division and integration mutual-rebirth together evolve, "First person one composite concepts -" "-〔N ( $1 \backslash \mathrm{n} 、 0)]$ of triple proportion immortal equal amount renew and natural number sets change spin system"; At same time sync vice versa. (Details check out "Theorem two Section 1").

"Whole corresponding, consubstantiality relative" as: Whole In naturally corresponding "First person one composite concepts - "-" itself sync compound immortal-renew division and integration mutual-rebirth evolve pace, with "First person one composite concepts -" "- $[N(1 \backslash n, 0)]$ of triple proportion immortal equal amount renew and natural number sets change spin system" consubstantiality relative. The following text similarly.

Based on "the proportion immortal equal amount rebirth-renew", Mathematical need to know, "一 natural completely number" is universe only one that having "Natural completely connotation change number set N" , but its own is "natural completely no change number". So "一" and "N" both are same one-completely-song played in different tone, "N" is "- ". 
So "Number always implication amount", vice versa. The following text similarly.】

Above same one song played in different tones as Religion:

Bible "Trinity of God". Tantric "Male Buddha Female Buddha coincide of Three Kayas completely Buddha". Buddhist scriptures "Buddha Dharma".

Please check out "Trinity" with "Theorem three" for "Trinity" compound corresponding multiple ways.

Above same one song played in different tones as Philosophy:

West needs to improve as "一's triple completely compound Commonness", "一's triple completely compound Being”, "一's triple completely compound Unique Personality"; China Book of Changes "Yin Yang Ball of Tai Ji”.

Above same one song played in different tones as Physics:

Science needs to improve as "Wave-Particle Completely Duality"; "Particle Completely Spin".

Etc.

\section{Second person one of two composite concepts 二.A}

"From 'zero frequency type non permutation-combination-orderly link' sync rebirth-out all '1 (local) frequency type permutation combination orderly link', one-coincide sync consummate hold balanced $(\backslash)$ compound immortal-renew division and integration mutual-rebirth evolve frequency type permutation combination orderly body 1 -link $\mid n$-links".

Abbreviation:

"1-link \n-links of one-coincide sync consummate hold balanced $(\backslash)$ compound immortal-renew division and integration mutual-rebirth evolve frequency type permutation combination orderly body".

"1-link \n-links".

Or, "1\n". (This formula represents "二.A" all the formula).

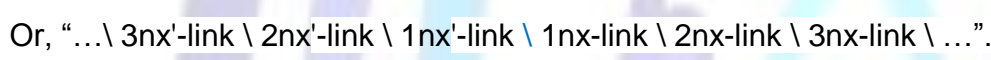

Or, “.. \3nx' $\backslash 2 n x^{\prime} \backslash 1 n x^{\prime} \backslash 1 n x \backslash 2 n x \backslash 3 n x \backslash \ldots ”$

Or, “..$\backslash 3 n x^{\prime}$-group-links $\backslash 2 n x^{\prime}$-group-links $\backslash 1 n x^{\prime}$-group-links $\backslash 1 n x$-group-links $\backslash 2 n x$-group-links $\backslash 3 n x-g r o u p-l i n k s ~ \backslash \ldots$.

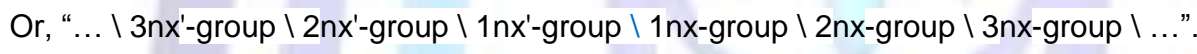

Etc.

【Above same one song played in different tone as mathematics:

"One-coincide sync consummate hold balanced $(\backslash)$ compound immortal-renew division and integration mutual-rebirth evolve, $1 / 2$ proportion equal amount renewed frequency type permutation combination orderly natural number sets change mathematical formula $1 \backslash n$ (its amount composite correspond conservation-equal to 0 amount)".

Abbreviation: “ $1 / 2$ proportion immortal equal amount renew and natural number sets change mathematical formula $1 \backslash n$ ”. Or same as above.

In other words, exclude mathematical "Second person one of two composite concepts 二.B" "0 natural number" with its amount, "Third and fourth persons three composite concepts" all "1 (local) natural number" with each amount consummate hold balanced $(\backslash)$ will be "Second person one of two composite concepts 二. $A$ " " $1 / 2$ proportion immortal equal amount renew and natural number sets change mathematical formula 〔(1-natural-number with its amount life and death change) $\backslash(\mathrm{n}$ number-set with its amount life and death change) ]".

"Second person one of two composite concepts 二.A" " $1 \backslash n$ " is "First person one composite concepts -" “— $[N(1 \backslash n, 0)]$ " itself, at same one position coincide together, " $1 / 2$ proportion immortal equal amount renew and natural number sets change composition's body".

\{ Second person one of two composite concepts 二.A" " $1 / 2$ proportion immortal equal amount renew and natural number sets change mathematical formula 〔(1-natural-number with its amount life and death change) \(n-number-set with its amount life and death change) ] "\}, and, ("Second person one of two composite concepts 二.B" " $1 / 2$ proportion immortal equal amount renew and 0 natural number never change"), at exactly same one position, whole corresponding, consubstantiality relative, sync compound immortal-renew division and integration mutual-rebirth equal amount exchange evolve become each other. This is the reason why "Second person one of two composite concepts 二. $A$ " " $1 / 2$ proportion immortal equal amount renew and natural number sets change composition's body" is the mathematical formula (its amount equal to 0$)$.

Combine with above e.g. solid ball, in "Third and fourth persons three composite concepts", Humanity or group or personal, from each own position, angles interface, function etc. show and unfold to "Second person one of two composite concepts 二.A" "mathematical formula $1 \backslash n$ ", they are all same one song played in different tones.

Combine with 二.3 of Theorem Two, in the category of "Second person one of two composite concepts 二.A", "Third and fourth persons three composite concepts" mathematics all "1 (local) natural number" could be unlimited division structures, 
the "amount". 】

but if they are combined with physical "amount" to calculate it, then people need to find ways not double counting

Above same one song played in different tone as Religion:

Bible "Moral consummate hold balanced Holy Body of Trinity—could be unlimited division Holy Bread"; Tantric "Male Buddha of Three Kayas completely Buddha".

Above same one song played in different tone as Philosophy:

Western needs to improves as "All local individuals that consummate hold balance personality together booming of onecoincide Commonness"; "consummate hold balance of one-coincide Unique Personality". China Book of Changes "Yang Yi of Yin Yang Ball of Tai Ji" or "Consummate hold balance the eight trigrams of Yin Yang Ball of Tai Ji" until "consummate hold balance the yin and the yang unlimited corresponding divinatory symbols of Yin Yang Ball of Tai Ji".

Above same one song played in different tone as Physical:

Science needs to improve as "All local time and space particles consummate hold -balanced together booming order of Tension-equation $11 \mathrm{n}$ " (Its amount composite correspond conservation-equal to "Second person one of two composite concepts 二.B" "Zero time and space Gravitational Primitives-Field amount"). "Wave-Particle Consummate Hold Balanced Duality".

Etc.

In "Second person one of two composite concepts 二.A":

Blue symbols \ representative: Whole corresponding, consubstantial relative, sync one-coincide consummate hold balanced compound (immortal and have-life-have-death)-renew division and integration mutual-rebirth evolve.

Black symbols \ representative: Whole corresponding, consubstantial relative, sync one-coincide consummate hold balanced compound (have-life-have-death)-renew division and integration mutual-rebirth evolve.

Symbols ... representative: et cetera reach to "Second person one of two composite concepts 二.A" " $1 \backslash n$ ".

\section{Second person one of two composite concepts 二.B}

"Consummate hold balanced all '1 (local) frequency type permutation combination orderly link' of $1 \backslash \mathrm{n}$, sync perfect evenly melt compound immortal-renew division and integration mutual-rebirth evolve zero frequency type non permutationcombination-orderly link"

Abbreviation:
"Zero-link".
Or, "Zero".
Or, "0".
.....

【 Above same one song played in different tones as mathematics:

"One-coincide sync perfect evenly melt compound immortal-renew division and integration mutual-rebirth evolve, $1 / 2$ proportion equal amount renew zero frequency type non permutation-combination-orderly natural number never change (its amount composite correspond conservation-equal to 1 ln amount)".

Abbreviation: " $1 / 2$ proportion immortal equal amount renew and 0 natural number never change link". Or same as above.

So, "Second person one of two composite concepts 二.A" is unity of "mathematics zero-natural-number and the amount of physical”.

"Second person one of two composite concepts 二.B" " 0 " is "First person one composite concepts -" “— $(N(1 \backslash n 、 0)]$ " itself, at same one position coincide together, other " $1 / 2$ proportion immortal equal amount renew and natural number never change composition's body".

["Second person one of two composite concepts 二.A" " $1 / 2$ proportion immortal equal amount renew and natural number sets change mathematical formula〔(1-natural-number with its amount have life have death change) \(n-number-set with its amount have life have death change) ] "\}, and, ("Second person one of two composite concepts 二.B" " $1 / 2$ proportion immortal equal amount renew and 0 natural number never change"), at exactly same one position, whole corresponding, consubstantial relative, sync compound immortal-renew division and integration mutual-rebirth equal amount exchange evolve become each other. This is why the mathematics blurred use "equal sign" between them still established.

Based on " $1 / 2$ proportion immortal equal amount rebirth-renew", Mathematical need to know, "Second person one of two composite concepts 二.B" "0 natural number" is universe only one that doesn't having "Natural number set" of "natural perfect evenly melt never change number"; But "Second person one of two composite concepts 二.B" "0 natural perfect evenly melt never change number-link" is "First person one composite concepts - " - $[N(1 \backslash n, 0)\rfloor$ " itself link of structures. 
"Second person one of two composite concepts 二.A" "Positive and negative natural number with each own amount sequence, consummate hold balanced '..$\backslash 3 n x^{\prime} \backslash 2 n x^{\prime} \backslash 1 n x^{\prime} \backslash 1 n x \backslash 2 n x \backslash 3 n x \backslash \ldots$. ", Combine with "Theorem three": So "Second person one of two composite concepts 二.B" "0" isn't dividing-demarcation-line of "Third and fourth persons three composite concepts" all "positive and negative natural number"; "Persons-concepts, Position, Trend", these three key elements, jointly determine "Third and fourth persons three composite concepts" all "1 natural number", sync compound relative to keep (and, or) conversion each self or each other positive and negative natural's standpoint and attributes. 】

Above same one song played in different tones as Religion:

Bible "Holy Ghost of Trinity--Perfectly understanding of soul"; Tantric "Female Buddha of Three Kayas completely Buddha".

Above same one song played in different tones as Philosophy:

Western needs to improves as "Perfect evenly melt of one-coincide Commonness"; "Perfect evenly melt of one-coincide Unique Personality". China Book of Changes "Yin Yi of Yin Yang Ball of Tai Ji".

Above same one song played in different tone as Physical:

Science needs to improve as "Zero time and space Gravitational-Primitives-Field (Its amount composite correspond conservation-equal to "Second person one of two composite concepts 二.A" "All local time and space particles consummate hold -balanced together booming order of Tension-equation $1 \backslash n$ amount).

"Second person one of two composite concepts 二.B" "Zero" is below "Third and fourth persons three composite concepts" all "1 local particle", consummate hold balance of "Second person one of two composite concepts 二.A" "1-particlelnparticles" at same time, perfect evenly melt become "zero commonness memory", this is why "Zero" also call "ZeroPrimitives"; "Zero" itself no structures, and even it "immortal-rebirth" from "frequency" of "First person one composite concepts - " and "Second person one of two composite concepts 二.A", but immortal life of "Zero no displacement' as "and 二.A", the "frequency" only "immortal equal renew Zero's amount". And "Root of perfect evenly melt zero commonness memory" rooted at "First person one composite concepts - " - $[N(1 \backslash n, 0)]$ of triple completely compound spin foundation-system\}".

Etc.

\section{Third person one composite concepts 三}

"1〔 n (nx1-group-links series、nx'1-link series) 〕 of triple sync approximately compound (have life have death)-renew division and integration mutual-rebirth evolve frequency type permutation-combination orderly spin link.

\section{Abbreviation:}

"1〔 n (nx1-group-links series、 nx'1-link series) ] of triple approximately compound spin link".

Or, "1〔n(nx1-group series、nx'1-link series) 〕".

Or, "1〔 $n$ ( $n \times 1$-group series、 $n x ' 1$ series) 〕”.

Or, "1 frequency type permutation-combination orderly link".

Or , "1 (nx1-group、nx'1)".

Or, "1".

Etc.

【Above same one song played in different tones as mathematics:

" $1\{\mathrm{n} 〔 1 / 2$ proportion probability approximately equal amount (have life have death)- renew and natural number sets change $n x$-group series, $1 / 2$ proportion probability approximately equal amount (have life have death)-renew and natural number change nx'-link series ] of triple sync approximately compound (have life have death)-renew division and integration mutual-rebirth evolve proportion probability approximately equal amount renew frequency type permutationcombination orderly natural number-set mutative spin link\}".

Abbreviation: "1[n ( $\mathrm{nx} 1$-group series、 $n x^{\prime} 1$ series) 〕 of triple sync approximately compound (have life have death)renew division and integration mutual-rebirth evolve proportion probability approximately equal amount renew frequency type permutation-combination orderly natural number-set mutative spin link” ; or, "1〔 $n$ ( $n \times 1$-group series、nx'1 series) ] of triple proportion probability (have life have death) approximately equal amount renew and natural number-set change spin link"; or same as above.

So, "Third person one composite concepts 三” is unity of "mathematics 1-natural-number and the amount of physical".

"Third person one composite concepts 三” any "1" is mathematics exclude "Second person one of two composite concepts 二.B" "0 natural number", that "natural number"; or "1 ( nx1-group、 nx'1) of natural number-set of equation"; or "1 ( $\mathrm{nx1-}$ 


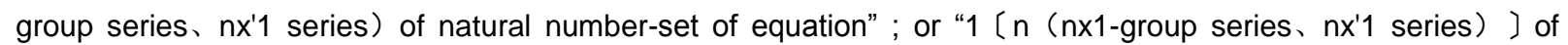
natural number-set of equation".

“Third person one composite concepts 三” its “1 of triple" composite correspond "Fourth person two composite concepts 四 .A、四.B" each "one coincide" establish; at same time vice versa. Known one or two of them, also need to know other one or two of them; otherwise human certification cognition always unable to have understand and balance syncretic fully inclusive.

〔 "Fourth person one of two composite concepts 四.A" “ $1 / 2$ proportion probability approximately equal amount (have life have death)-renew and natural number sets change nx-group" 〕, and, “Fourth person one of two composite concepts 四 .B" “ $1 / 2$ proportion probability approximately equal amount (have life have death)-renew and natural number change $n x^{\prime \prime \prime}$ ] , at approximately same one position, whole corresponding, consubstantiality relative, sync approximately compound (have life have death)-renew division and integration mutual-rebirth evolve each other at same time, at approximately same one position, sync approximately compound (have life have death)-renew division and integration mutual-rebirth together evolve, "Third person one composite concepts 三” “1〔 $n$ ( $n \times 1$-group series、 nx'1 series) 〕 of triple proportion probability (have life have death) approximately equal amount renew and natural number-set change spin link"; At same time sync vice versa. (Details check out 二.3 of Theorem two).

Among them, this or that "n of natural number sets with its amount", or "n of natural numbers with its all amount", or " $₫$ 〔 $1 / 2$ proportion probability approximately equal amount (have life have death)- renew and natural number sets change nxgroup series, $1 / 2$ proportion probability approximately equal amount (have life have death)-renew and natural number change nx'-link series ] ", all is same one song played in different tones as abbreviation this or that "1", departure from their own position property, overall corresponding, consubstantial relative, sync compound (have life have death)-renew division and integration mutual-rebirth evolve arrive to, their " $1 / 2$ proportion immortal equal amount renew and natural number sets change of consummate hold balanced composition body"--"Second person one of two composite concepts 二 .A" "1 $\backslash n$ " of position property, that "proportion probability approximately equal amount (have life have death)- renew development of natural number sets change n"; "Second person one of two composite concepts 二.B" is this or that " 1 " itself, other " $1 / 2$ proportion immortal equal amount renew and 0 natural number never change of perfect evenly melt composition body"; "First person one composite concepts -" is this or that "1" itself , " $-[N(1 \backslash n, 0)]$ of triple proportion equal amount renew and natural number-set change of completely composition body"; at same time on the contrary, "Third person one composite concepts 三" any "1", or "Third and fourth persons three composite concepts" all "1" , all is "First and Second person three composite concepts" each "1 proportion probability have life have death approximately equal amount renew and natural number change of local composition link". But "Third and fourth persons three composite concepts" all "1" each own expand to "First and Second person three composite concepts" each will be "1 [n (nx1-group series, $n x^{\prime} 1$ series) ]".

Above complete expression patterns as " $1\lceil n$ ( $n \times 1$-group series、nx'1 series) $) \leftrightarrow 一(N(1 \backslash n 、 0))$ ”, or "1〔n(nx1group series、 $n x^{\prime} 1$ series $\left.)\right] \ldots-(N(1 \backslash n, 0))$ ". Among them, symbol $\leftrightarrow$ representative: "Whole corresponding, consubstantial relative, sync compound (immortal、immortal with have-life-have-death 、 have-life-have-death)-renew division and integration mutual-rebirth evolve each own and each other". In here representative: "Whole corresponding, consubstantial relative, sync compound (have-life-have-death and immortal)-renew division and integration mutual-rebirth evolve each other".

So, "Third person one composite concepts 三” "1 [ n (nx1-group series、nx'1 series) 〕 " formula looks like "First person one composite concepts - "- $(N(1 \backslash n, 0))$ ", but this or that "1" not is this or that " $n$ ". this or that "1" synchronization in this or that "nx1-group series nx'1 series" development and expand growing, that is why "1" is 1-window's own department of "1 [ $n$ ( $n \times 1$-group series $n \times 1$ series) ]".

Combine with Theorem Three, "Third person one composite concepts 三” any "1〔 $n$ ( $n x 1$-group series、 $n x$ '1 series) ) natural number sets with amount", all keep their own position property at same time, change to position property of ["Fourth person one of two composite concepts 四.A" in "nx-group natural number sets with amount" any " 1 natural number with amount", and, "Fourth person one of two composite concepts 四.B" "nx' natural number with amount" $;$; at same time, vice versa.】

Above same one song played in different tones as Religion:

Bible "Son of Trinity"; Tantric "Female Buddha of Three Kayas Approximately Buddha".

Above same one song played in different tones as west Philosophy:

Western needs to improves as "1's triple approximately compound Commonness"; "1's triple approximately compound Unique Personality"; China Book of Changes "'The yin and the yang unlimited corresponding divinatory symbols' each own of Yin Yang Ball of Tai Ji".

Above same one song played in different tones as Physical:

Science needs to improve as "Wave-Particle Local Duality"; "Particle Local Spin".

Etc.

Fourth person one of two composite concepts 四.A 
"From ('zero frequency type non permutation-combination-orderly link', and, 'frequency type permutationcombination-orderly $n x^{\prime}$-link') sync rebirth-out all '1 (local) frequency type permutation combination orderly link', onecoincide sync approximately hold balanced $(\backslash$ compound (have life have death)-renew division and integration mutualrebirth evolve frequency type permutation combination orderly body nx-group" series.

Abbreviation:

"nx-group of one-coincide sync approximately hold balanced ( $\backslash$ compound (have life have death)-renew division and integration mutual-rebirth evolve frequency type permutation combination orderly body" series.

Or. "nx-group-links" series.

Or, "nx-group" series.

Or, "nx-group".

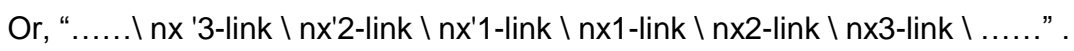

Or, “..... $n x x^{\prime} 3 \backslash n x^{\prime} 2 \backslash n x^{\prime} 1 \backslash n \times 1 \backslash n \times 2 \backslash n \times 3 \backslash \ldots . . "$

Or, “..... $n^{\prime} x^{\prime} 3$-group-links $\backslash n x^{\prime} 2$-group-links $\backslash n x^{\prime} 1$-group-links $\backslash n \times 1$-group-links $\backslash n x 2$-group-links $\backslash n \times 3$-group-links $\backslash \ldots \ldots$....

Or, “...... nx'3-group \nx'2-group \nx'1-group \nx1-group \nx2-group \nx3-group \....." .

Etc.

【Above same one song played in different tones as Mathematics:

"One-coincide sync approximately hold balanced $(\backslash)$ compound (have life have death)-renew division and integration mutual-rebirth evolve, $1 / 2$ proportion probability approximately equal amount (have life have death)-renew frequency type permutation combination orderly natural number sets change nx-group" series (its amount composite correspond approximately equal to $\mathrm{nx}^{\prime}$ series amount)".

\section{Abbreviation:}

“ $1 / 2$ proportion probability (have life have death) approximately equal amount renew and natural number sets change nxgroup" series. Or same as above.

So, "Fourth person one of two composite concepts 四.A" is unity of "nx-group natural-number set and the amount of physical".

"Fourth person one of two composite concepts 四.A" any "nx-group" is mathematics exclude "Second person one of two composite concepts 二.B" "0 natural number", that "nx-group natural number-set of equation"; or "nx-group natural number-set series of equation" ; or "n(nx-group natural number-set series of equation)".

"Fourth person one of two composite concepts 四.A" “nx-group" is “Third person one composite concepts 三” “1〔 $n$ (nx1group series $n x^{\prime} 1$ series) ] " itself, at same one position approximately coincide together, " $1 / 2$ proportion probability approximately equal amount (have life have death)-renew and natural number sets change composition's body".

〔 "Fourth person one of two composite concepts 四.A" " $1 / 2$ proportion probability approximately equal amount (have life have death)-renew and natural number sets change nx-group" 〕, and, ‘Fourth person one of two composite concepts 四 .B" " $1 / 2$ proportion probability approximately equal amount (have life have death)-renew and natural number change $n x "$ " ] , whole corresponding, consubstantiality relative, at approximately same one position, sync approximately compound (have life have death)-renew division and integration mutual-rebirth approximately equal amount exchange evolve become each other.

Combine with Theorem Three, in "Fourth person one of two composite concepts 四.A" "nx-group natural number sets with amount" any " 1 natural number with amount", all keep their own position property at same time, change to position property of ["Third person one composite concepts 三” "1〔 $n$ ( $n \times 1$-group series、nx'1 series) ] natural number sets with amount", and, "Fourth person one of two composite concepts 四.B" "nx' natural number with amount"\}; at same time, vice versa.】

Above same one song played in different tone as Religion:

Bible "Moral approximately hold balanced Body of Trinity"; Tantric "Male Buddha of Three Kayas Approximately Buddha".

Above same one song played in different tone as Philosophy:

Western needs to improves as "Group local individuals approximately hold balance personality together booming of onecoincide Commonness"; "Approximately hold balance of one-coincide Unique Personality". China Book of Changes "Yang of Yin Yang Ball of Trigram".

Above same one song played in different tone as Physical:

Science needs to improve as "Group local time and space particles approximately hold -balanced together booming order of Tension-equation nx-group" (Its amount composite correspond approximately-equal to "Fourth person one of two composite concepts 四.B" "nx' time and space Gravitational Particle-Field amount"). "Wave-Particle Approximately Hold Balanced Duality". 
Etc.

\section{Fourth person one of two composite concepts 四.B}

"Approximately hold balanced every '1 (local) frequency type permutation combination orderly link' of nx-group, onecoincide sync approximately evenly melt compound (have life have death)-renew division and integration mutual-rebirth evolve frequency type permutation combination orderly nx'-link" series.

\section{Abbreviation: \\ "nx'-link" series. \\ Or, "nx"' series. \\ Or, "nx"'.}

【Above same one song played in different tones as Mathematics:

"One-coincide sync approximately evenly melt compound (have life have death)-renew division and integration mutualrebirth evolve, $1 / 2$ proportion probability approximately equal amount (have life have death)-renew frequency type permutation combination orderly natural number change $n x^{\prime}$ link" series (its amount composite correspond approximately equal to nx-group series amount)".

\section{Abbreviation:}

“ $1 / 2$ proportion probability (have life have death) approximately equal amount renew and natural number change nx' link" series. Or same as above.

So, "Fourth person one of two composite concepts 四.B" is unity of "nx-group natural-number and the amount of physical".

"Fourth person one of two composite concepts 四.B" any "nx"' is mathematics exclude "Second person one of two composite concepts 二.B" "0 natural number", that "natural number".

"Fourth person one of two composite concepts 四.B" "nx"' is "Third person one composite concepts 三” “1〔 n (nx1-group series $n x^{\prime} 1$ series) ] of natural number set with amount" itself, at same one position approximately coincide together, other " $1 / 2$ proportion probability approximately equal amount (have life have death)-renew and natural number change composition's body".

〔 "Fourth person one of two composite concepts 四.A" “ $1 / 2$ proportion probability approximately equal amount (have life have death)-renew and natural number sets change nx-group"], and, ["Fourth person one of two composite concepts 四 .B" " $1 / 2$ proportion probability approximately equal amount (have life have death)-renew and natural number change nx"' ] , whole corresponding, consubstantiality relative, at approximately same one position, sync approximately compound (have life have death)-renew division and integration mutual-rebirth approximately equal amount exchange evolve become each other. This is the way from "Structure showing the amount nx-group" of "Fourth person one of two composite concepts 四 .A", approximately equal inferred, "Structure hard showing the amount" of "Fourth person one of two composite concepts 四.B" "approximately uniform melt nx"”.

Combine with Theorem Three, "Fourth person one of two composite concepts 四.B" any "nx' natural number with amount " , all keep their own position property at same time, change to position property of \{ "Third person one composite concepts 三” "1〔 $n$ ( $n \times 1$-group series $n x ' 1$ series) 〕 natural number set with amount", and, "Fourth person one of two composite concepts 四.A" in "nx-group natural number set with amount" any "1 natural number with amount" \}; at same time, vice versa.】

Above same one song played in different tones as Religion:

Bible "Soul approximately evenly melt understanding of Trinity"; Tantric "Female Buddha of Three Kayas Approximately Buddha".

Above same one song played in different tone as Philosophy:

Western needs to improves as "Approximately evenly melt of one-coincide Commonness"; "Approximately evenly melt of one-coincide Unique Personality". China Book of Changes "Yin of Yin Yang Ball of Trigram".

Above same one song played in different tone as Physical:

Science needs to improve as "nx' time and space Gravitational Particle-Field amount" (Its amount composite correspond approximately-equal to "Fourth person one of two composite concepts 四.A" "Group local time and space particles approximately hold-balanced together booming order of Tension-equation nx-group amount"). "Wave-Particle Approximately Evenly Melt Duality".

Etc.

\section{Six composite concepts of Quadrity (Inclusive human all the cognition and certification)}




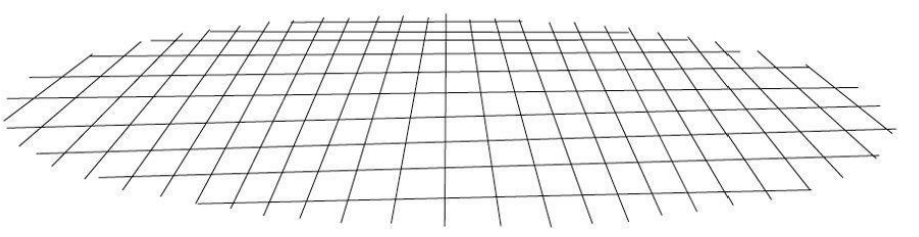

宇宙生命基础位格二.A、四. A

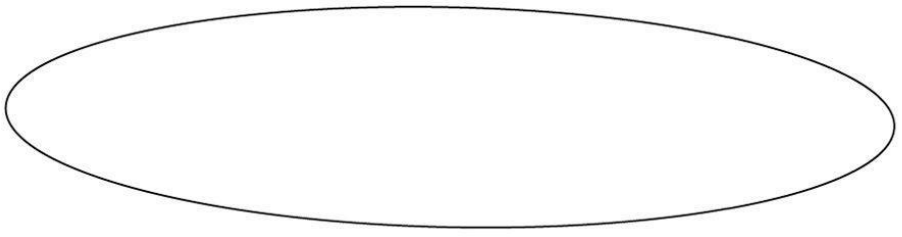

宇宙生命基础位格二. B、四. B

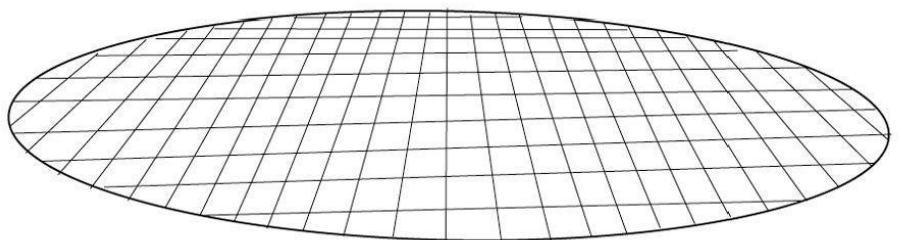

宇宙生命基础位格一、三

\section{Section 2. Do not from human function formatting "Complete Universe"}

Religion: Quran and Bible both had "Allah invisible" and Buddhist scriptures "Nirvana".

Above same one song played in different tone as Philosophy:

West "Nothingness". China Book of Changes "Wu Chi" of Philosophy.

Etc.

\section{Section 3. "Complete universe's own department of Trinity"}

"Theorem one.1. and Theorem one. 2." combine with "Theorem three",

First-Person: "Basis's own department" 《The principle of natural law》 "Complete-Universe", it has same one song played in different tones as, (Bible "God" and "Trinity" and "cross"; Buddhist scriptures "Buddha", Tantric "Three Kayas Buddha"; Western "Noumenon"; The Book of Changes "Yin Yang Ball"; Quran "Allah"; Tao Te Ching "Tao" etc.) composite correspond show and unfold:

Second-Person: From human function formatting 《The principle of natural law》(Structured) "First person one composite concepts - "- $[N(1 \backslash n, 0)]$ of triple completely compound spin system", it has same one song played in different tones as 〔Bible "God of moral tangible system", "Trinity of Father, Son, Holy Spirit (and Holy Body)", "One vertical of Cross"; Buddhist scriptures "Buddha-Dharma"; Tantric "Male Buddha of Three Kayas completely Buddha"; Western "Being"; The Book of Changes "Tai Chi of Yin Yang Ball", "Yang of Yin Yang Ball"; Quran "(Man white dress to show) Moral tangible system of Allah"; Tao Te Ching "Tao Law" etc. ] (which is Theorem one.1.).

And,

Third-Person: Not from human function formatting 《The principle of natural law》 "No human language could described Complete-Universe", it has same one song played in different tones as〔Quran "(Woman black dress to show) Allah invisible" ; Bible "God invisible"; Buddhist scriptures "Nirvana", Tantric "Female Buddha of Three Kayas completely Buddha"; Western "Nothingness"; The Book of Changes "Wu Chi", "Yin of Yin Yang Ball"; Tao Te Ching "Nothing" etc. ] (which is Theorem one.2.).

Above three persons composite correspond establish Trinity. Among them, "Second person" and "Third-Person" both are "First-Person" its own showed in different ways. These three persons are each other and as one.

Among them, due to lack of many of support, such as physical amount etc. Mathematics positive numbers and negative numbers did not grow to "First person one composite concepts" "- [N (1-link\n-links、0-link) ] of triple completely compound spin system" its own and its own dual trend of division-positive and integration-negative yet; Naturally there will be no growth to "Complete universe's own department of trinity" its own and its composite correspond standpoint and attributes as "Tangible-positive-number and Invisible-negative-number". 
Western philosophy "Ontology" and science quantum theory same as above, they are both didn't know that "No human language could described 'Nothingness"' with (unstructured "Second person one of two composite concepts 二.B" "Zero link") are different things also. They are both need have long way to establish a sound cognition and certification.

"Theorem three" will be comprehensive carding clear Sciences "Relativistic", philosophy and religion "Dialectics".

\section{Theorem Two: "Basis on 'synchronous multi-D mutual-birth generate frequency, synchronous multi-D mutual-rebirth generate frequency cycle', Principle show and unfold in six composite concepts Quadrity, sync compound (immortal, immortal with have-life-have-death, have-life-have-death)-renew division and integration mutual- rebirth evolve each own and mutual presence ways and presence relationships and build in Complete mathematical modeling"}

Section 1. 《The principle of natural law》work with overall.

Basis on the "Theorem one. Section 3. Second-Person", which is "First person one composite concepts - " - 〔 N (1link ln-links、0-link) ] of triple completely compound spin system" itself dual trend of division and integration, "Theorem two" use "abbreviation symbol" to help people see clear principle.

\section{Principle model e.g.1.}

〔 "Third and Fourth persons three composite concepts" all "1", or same one song played in different tones as ("Third persons one composite concepts 三” all "1", "Fourth persons one of two composite concepts 四.A" all "nx-group", "Fourth persons one of two composite concepts 四.B" all "nx' "), or same one song played in different tones as etc. J, in naturally corresponding follow tend to "First and Second persons three composite concepts" respective standpoint and attributes at same time, departure from (三、四.A、四.B) their respective "position, angle. interface" standpoint and attributes, Omni-dimensional inside and outside, whole corresponding, consubstantiality relative, sync deep-broad (approximately or) compound \{have life have death〔(have life have death) and immortal〕 \}-renew, division and integration mutual-rebirth evolve to,

\{ "Second person one of two composite concepts 二.A" [ ‘ "Third and Fourth persons three composite concepts" all "1", or same one song played in different tones as ("Third persons one composite concepts 三” all "1", "Fourth persons one of two composite concepts 四.A" all "nx-group", "Fourth person one of two composite concepts 四.B" all "nx' "), or same one song played in different tones as etc. ] respective, whole corresponding, consubstantiality relative, from "Second person one of two composite concepts 二.B" "0" sync compound rebirth-out, at category of "Second persons one of two composite concepts 二.A", sync approximately compound (have life have death)-renew division and integration mutualrebirth evolve respective and each other at same time, one-coincide sync consummate hold balanced $(\backslash)$ compound immortal-renew division and integration mutual-rebirth evolve $1 \backslash n$ " ], and, "Second person one of two composite concepts 二.B" [ "Second persons one of two composite concepts 二.A" "Consummate hold balanced〔 'Third and Fourth persons three composite concepts' all "1", or same one song played in different tones as ("Third persons one composite concepts 三” all "1", "Fourth persons one of two composite concepts 四.A" all "nx-group", "Fourth persons one of two composite concepts 四.B" all "nx' "), or same one song played in different tones as etc. ], sync perfect evenly melt compound immortal-renew division and integration mutual-rebirth evolve 0"] \} respective;

At same time, "Second person one of two composite concepts 二.A" "1 \n" and "Second person one of two composite concepts 二.B" "0", whole in naturally corresponding "First person one composite concept $\mathrm{S}$ 一" "一" itself sync compound immortal-renew division and integration mutual-rebirth evolve pace, with "First person one composite concepts - " - $〔 \mathrm{~N}$

$(1 \backslash n, 0)$ ] " consubstantiality relative, at exactly same one position, sync compound immortal-renew division and integration mutual-rebirth (equal amount exchange) evolve each other at same time, at exactly same one position, sync compound immortal-renew division and integration mutual-rebirth (equal amount) together evolve,

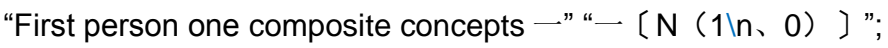

At same time sync on the contrary,

"First person one composite concepts -" "- $[N(1 \backslash n, 0)]$ ", in naturally corresponding follow tend to [Third and Fourth persons three composite concepts" all "1", or same one song played in different tones as ("Third persons one composite concepts 三” all "1", "Fourth persons one of two composite concepts 四.A" "nx-group", "Fourth person one of two composite concepts 四.B" " $n \mathbf{x}^{\prime}$ "), or same one song played in different tones as etc. ] each standpoint and attributes at same time, departure from "- " its own standpoint and attributes, also its own whole corresponding, consubstantiality relative, sync gather to compound immortal-renew division and integration mutual-rebirth evolve to, "Second person one of two composite concepts 二.A" "1 1 n" and "Second person one of two composite concepts 二.B" "0" respective;

At same time, "Second person one of two composite concepts 二.A" "1 \n" and "Second person one of two composite concepts 二.B" "0", whole in naturally corresponding "First person one composite concept $\mathrm{s}$-" "一" itself sync compound immortal-renew division and integration mutual-rebirth evolve pace, with "First person one composite concepts - " - $〔 \mathrm{~N}$ 
$(1 \backslash n, 0)$ ] " consubstantiality relative, at exactly the same one position, sync compound immortal-renew division and integration mutual-rebirth (equal-amount exchange) evolve each other at same time, sync gather to compound ‘ immortal, immortal and (have life have death) ] -renew division and integration mutual-rebirth evolve to,

〔 "Third and Fourth persons three composite concepts" all "1", or same one song played in different tones as ("Third persons one composite concepts 三” all "1", "Fourth persons one of two composite concepts 四.A" all "nx-group", "Fourth person one of two composite concepts 四.B" all "nx' "), or same one song played in different tones as etc. J respective;

At same time, [ "Third and Fourth persons three composite concepts" all "1", or same one song played in different tones as ("Third persons one composite concepts 三” all "1", "Fourth persons one of two composite concepts 四.A" all "nxgroup", "Fourth person one of two composite concepts 四.B" all "nx' "), or same one song played in different tones as etc. J respective, whole in naturally corresponding "First person one composite concepts -" "-" itself sync compound immortal-renew division and integration mutual-rebirth evolve pace, with "First person one composite concepts - "

$(1 \backslash n 、 0)$ ] " consubstantiality relative, from "Second person one of two composite concepts 二.B" "0" sync compound rebirth-out, at category of "Second persons one of two composite concepts 二.A" "1\n", sync gather to compound (have life have death)-renew division and integration mutual-rebirth evolve respective and each other;

Above same one song played in different tone as below:

Proportion immortal $\quad 1 / 2$ proportion immortal equal-amount renew and natural

Equal-amount renew

and - natural number

no change

number sets change mathematical formula $\mathbf{1} \backslash \mathbf{n}$

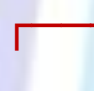

$1 / 2$ proportion immortal equal-amount renew and 0 natural number no change triple proportion immortal

equal amount-renew and

natural number sets change

spin system $\mathbf{N}$

At same time sync vice versa.

\section{Principle model e.g.2.}

In "Third person one composite concepts 三” any of this or that $\{$ "1", or "1 ( $\mathrm{n}$ ( $\mathrm{nx} 1$-group series、 $\mathrm{nx}$ ' 1 series) ) " $\}$, in naturally corresponding follow tend to $\{$ "First person one composite concepts -" "一〔N ( 1 n、 0$)]$ ", "Second person one of two composite concepts 二.A" " $1 \backslash$ n" and "Second person one of two composite concepts 二.B" " 0 " \} respective standpoint and attributes at same time, departure from \{ "1", or "1 ( $n$ ( $n \times 1$-group series, $n x^{\prime} 1$ series) ) " its own "position, angle. interface" standpoint and attributes, Omni-dimensional inside and outside, whole corresponding, consubstantiality relative, at approximately same one position, sync deep-broad approximately compound (have life have death)-renew, division and integration mutual-rebirth evolve to, ("Fourth persons one of two composite concepts 四.A" any of this or that "nx1-group series", and, "Fourth person one of two composite concepts 四.B" any of this or that "nx' 1 series" ) respective;

At same time, "Fourth persons one of two composite concepts 四.A" any of this or that "nx1-group series", and, "Fourth person one of two composite concepts 四.B" any of this or that "nx' 1 series", whole in naturally corresponding "First person one composite concepts -" "-" itself sync compound immortal-renew division and integration mutual-rebirth evolve pace, with "First person one composite concepts - " $-[N(1 \backslash n, 0)]$ " consubstantiality relative, at approximately same one position, sync deep-broad approximately compound (have life have death)-renew division and integration mutual-rebirth (approximately equal amount exchange) evolve each other at same time, sync deep-broad compound [ have life have death, (have life have death) and immortal) -renew division and integration mutual-rebirth evolve to,

\{ "Second person one of two composite concepts 二.A" [ 〔 "Third person one composite concepts 三” any of this or that "1", and , ("Fourth persons one of two composite concepts 四.A" all in this or that "nx1-group" series every "1", "Fourth person one of two composite concepts 四. $B^{\prime}$ all of this or that "nx' $\mathbf{1}^{\prime}$ " series respective) together this or that " $n$ "] , whole corresponding, consubstantiality relative, from "Second person one of two composite concepts 二.B" "0" sync compound rebirth-out, at category of "Second persons one of two composite concepts 二.A", sync approximately compound (have life have death)-renew division and integration mutual-rebirth evolve respective and each other at same time, one-coincide sync consummate hold balanced ( $($ ) compound immortal-renew division and integration mutual-rebirth evolve $1 \backslash n$ " ], and, "Second person one of two composite concepts 二.B" [ "Second persons one of two composite concepts 二.A" "Consummate hold balanced "Third person one composite concepts 三” any of this or that "1", and , ( ("Fourth persons one of two composite concepts 四.A" all in this or that "nx1-group" series every "1", "Fourth person one of two composite concepts 四.B" all of this or that "nx' 1" series respective) together this or that " $n$ " ] , sync perfect evenly melt compound immortal-renew division and integration mutual-rebirth evolve 0" ] \} respective; 
At same time, "Second person one of two composite concepts 二.A" "1 $\backslash n$ " and "Second person one of two composite concepts 二.B" "0", whole in naturally corresponding "First person one composite concepts - "一" itself sync compound immortal-renew division and integration mutual-rebirth evolve pace, with "First person one composite concepts -" " $[N(1 \backslash n, 0)]$ " consubstantiality relative, at exactly same one position, sync compound immortal-renew division and integration mutual-rebirth (equal amount exchange) evolve each other at same time, at exactly same one position, sync compound immortal-renew division and integration mutual-rebirth (equal amount) together evolve,

"First person one composite concepts -" “〔 $[N(1 \backslash n, 0)] " ;$

At same time sync on the contrary,

"First person one composite concepts -" "- $[N(1 \backslash n, 0)]$ ", in naturally corresponding follow tend to ["Third person one composite concepts 三” any of this or that "1", and , ( "Fourth persons one of two composite concepts 四.A" all of this or that "nx1-group" series every "1", "Fourth person one of two composite concepts 四.B" all of this or that "nx' 1 " series respective) together this or that " $n$ " $〕\}$ respective standpoint and attributes at same time, departure from "一〔 $N$ ( $1 \backslash n, 0$ ) ] " its own standpoint and attributes, also its own whole corresponding, consubstantiality relative, sync gather to compound immortal-renew division and integration mutual-rebirth evolve to, ("Second person one of two composite concepts 二.A" "1 $\backslash$ n" and "Second person one of two composite concepts 二.B" "0" ) respective;

At same time, "Second person one of two composite concepts 二.A" "1 \n" and "Second person one of two composite concepts 二.B" "0", whole in naturally corresponding "First person one composite concepts —" "—" itself sync compound immortal-renew division and integration mutual-rebirth evolve pace, with "First person one composite concepts - " $-〔 \mathrm{~N}$

$(1 \backslash n, 0)$ ] " consubstantiality relative, at exactly the same one position, sync compound immortal-renew division and integration mutual-rebirth (equal-amount exchange) evolve each other at same time, sync gather to compound 〔immortal, immortal and (have life have death) ] -renew division and integration mutual-rebirth evolve to,

("Fourth persons one of two composite concepts 四.A" this or that "nx1-group series" , and, "Fourth person one of two composite concepts 四.B" this or that "nx' 1 series") respective;

At same time, ("Fourth persons one of two composite concepts 四.A" this or that "nx1-group series" , and, "Fourth person one of two composite concepts 四.B" this or that "nx' $\mathbf{1}$ series"), whole in naturally corresponding "First person one composite concepts - "- itself sync compound immortal-renew division and integration mutual-rebirth evolve pace, with "First person one composite concepts -" "- $[N(1 \backslash n, 0)]$ " consubstantiality relative, from "Second person one of two composite concepts 二.B" "0" sync compound rebirth-out, at category of "Second persons one of two composite concepts

二.A" “1 $\backslash n$ ", and (四.A and 四.B) approximately same one position, sync compound (have life have death)-renew division and integration mutual-rebirth (approximately equal amount exchange) evolve each other at same time, sync gather to compound (have life have death)-renew division and integration mutual-rebirth (approximately equal amount) together evolve,

"Third person one composite concepts 三” any of this or that $\left\{\right.$ "1", or “ 1 ( $n$ ( $n \times 1$-group series、 $n \mathbf{x}^{\prime} 1$ series) ) "\};

Above same one song played in different tone as below:

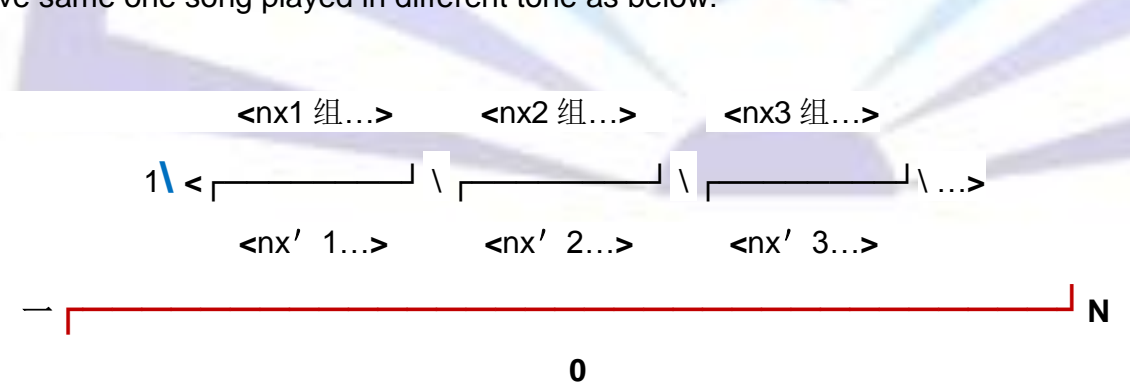

Above same one song played in different tone as below:

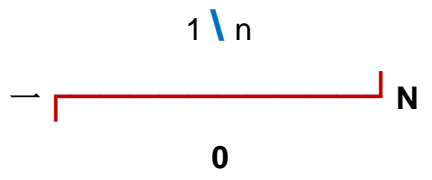

At same time sync vice versa. 
Attached:

Universal Unified Field System of Science, Particle Perfect Spin System of Science Similarly with above.

\section{Principle model e.g.3.}

"Fourth persons one of two composite concepts 四.A" any of this or that "nx-group", in naturally corresponding follow tend to $\left\{\right.$ "First person one composite concepts -" "-〔 $\left[N\left(\ldots \mid 3 n x^{\prime} \backslash 2 n x^{\prime} \backslash 1 n x^{\prime} \backslash 1 n x \backslash 2 n x \backslash 3 n x \backslash \ldots 0\right)\right]$ ”, "Second person one of two composite concepts 二.A" "... $\mid 3 n x^{\prime} \backslash 2 n x^{\prime} \backslash 1 n x^{\prime} \backslash 1 n x \backslash 2 n x \backslash 3 n x \backslash \ldots$. and "Second person one of two composite concepts 二.B" "0" \} respective standpoint and attributes at same time, departure from "nx-group" its own "position, angle. interface" standpoint and attributes, Omni-dimensional inside and outside, whole corresponding, consubstantiality relative, sync deep-broad approximately hold balanced compound (have life have death)-renew, division and integration mutual-rebirth evolve to, ("Fourth persons one of two composite concepts 四.A" (any of this or that " $\mathrm{nx} 1$ group series", and, any of this or that "nx' 1-group series" ) respective;

At same time, "Fourth persons one of two composite concepts 四.A" (any of this or that "nx1-group series", and, any of this or that " $n x^{\prime}$ 1-group series"), whole in naturally corresponding "First person one composite concept s -" "一" itself sync compound immortal-renew division and integration mutual-rebirth evolve pace, with "First person one composite concepts -" "- $\left[N\right.$ ( “... $\left.\left.\mid 3 n x^{\prime} \backslash 2 n x^{\prime} \backslash 1 n x^{\prime} \backslash 1 n x \backslash 2 n x \backslash 3 n x \backslash \ldots, 0\right)\right]$ ” consubstantiality relative, sync deep-broad approximately hold balanced compound (have life have death)-renew division and integration mutual-rebirth evolve each other at same time, sync deep-broad (approximately or) compound ( have life have death, (have life have death) and immortal] -renew division and integration mutual-rebirth evolve to,

["Second person one of two composite concepts 二.A" [ 〔 "Third and Fourth persons three composite concepts" (all of this or that "nx1-group" series, and, all of this or that "nx' 1-group" series)], whole corresponding, consubstantiality relative, from "Second person one of two composite concepts 二.B" "0" sync compound rebirth-out, at category of "Second persons one of two composite concepts 二.A", sync approximately hold balanced compound (have life have death)-renew division and integration mutual-rebirth evolve respective and each other at same time, one-coincide sync consummate hold balanced compound immortal-renew division and integration mutual-rebirth evolve '... $\mid 3 n x^{\prime} \backslash 2 n x^{\prime} \backslash 1 n x^{\prime} \backslash 1 n x \backslash$ $2 n x \backslash 3 n x \backslash . . . "$ " ], and, "Second person one of two composite concepts 二.B" [ "Second persons one of two composite concepts 二.A" [ "Consummate hold balanced "Third and Fourth persons three composite concepts" (all of this or that "nx1-group" series, and, all of this or that "nx' 1-group" series)], sync perfect evenly melt compound immortal-renew division and integration mutual-rebirth evolve 0" ] \} respective;

At same time, "Second person one of two composite concepts 二.A" “... $3 n x^{\prime} \backslash 2 n x^{\prime} \backslash 1 n x^{\prime} \backslash 1 n x \backslash 2 n x \backslash 3 n x \backslash . .$. " and "Second person one of two composite concepts 二.B" " 0 ", whole in naturally corresponding "First person one composite concepts -" "-" itself sync compound immortal-renew division and integration mutual-rebirth evolve pace, with "First person one composite concepts -" "- $\left[N\left(\ldots \mid 3 n x^{\prime} \backslash 2 n x^{\prime} \backslash 1 n x^{\prime} \backslash 1 n x \backslash 2 n x \backslash 3 n x \backslash \ldots 、 0\right)\right]$ " consubstantiality relative, at exactly same one position, sync compound immortal-renew division and integration mutual-rebirth (equal amount exchange) evolve each other at same time, at exactly same one position, sync compound immortal-renew division and integration mutual-rebirth (equal amount) together evolve,

"First person one composite concepts -" "—〔N ( .. $\left.\left.\mid 3 n x^{\prime} \backslash 2 n x^{\prime} \backslash 1 n x^{\prime} \backslash 1 n x \backslash 2 n x \backslash 3 n x \backslash \ldots, 0\right)\right]$;

At same time sync on the contrary,

"First person one composite concepts -" "—〔N (.. $\left.\left.\mid 3 n x^{\prime} \backslash 2 n x^{\prime} \backslash 1 n x^{\prime} \backslash 1 n x \backslash 2 n x \backslash 3 n x \backslash \ldots, 0\right)\right]$ ”, in naturally corresponding follow tend to "Fourth persons one of two composite concepts 四.A" any of this or that ("nx-group", or same one song played in different tone as "..... $\left.33 n x^{\prime} \backslash 2 n x^{\prime} \backslash 1 n x^{\prime} \backslash 1 n x \backslash 2 n x \backslash 3 n x \backslash \ldots . ..\right)$ respective standpoint and attributes at same time, departure from " - $\left[N\left(\ldots \backslash 3 n x^{\prime} \backslash 2 n x^{\prime} \backslash 1 n x^{\prime} \backslash 1 n x \backslash 2 n x \backslash 3 n x \backslash \ldots 、 0\right)\right]$ ” its own standpoint and attributes, also its own whole corresponding, consubstantiality relative, sync gather to compound immortal-renew division and integration mutual-rebirth evolve to, ("Second person one of two composite concepts 二.A" “... $\mid 3 n x^{\prime} \backslash 2 n x^{\prime} \backslash$ $1 n x^{\prime} \backslash 1 n x \backslash 2 n x \backslash 3 n x \backslash . . . "$ and "Second person one of two composite concepts 二.B" "0" ) respective;

At same time, "Second person one of two composite concepts 二.A" "... $3 n x^{\prime} \backslash 2 n x^{\prime} \backslash 1 n x^{\prime} \backslash 1 n x \backslash 2 n x \backslash 3 n x \backslash . .$. " and "Second person one of two composite concepts 二.B" "0", whole in naturally corresponding "First person one composite concepts -" "- itself sync compound immortal-renew division and integration mutual-rebirth evolve pace, with "First person one composite concepts -" "-〔N ( . $\left.\left.13 n x^{\prime} \backslash 2 n x^{\prime} \backslash 1 n x^{\prime} \backslash 1 n x \backslash 2 n x \backslash 3 n x \backslash \ldots 、 0\right)\right]$ " consubstantiality relative, at exactly the same one position, sync compound immortal-renew division and integration mutual-rebirth (equalamount exchange) evolve each other at same time, sync gather to compound 〔 immortal, immortal and (have life have death) ] -renew division and integration mutual-rebirth evolve to,

"Fourth persons one of two composite concepts 四.A" (any of this or that "nx1-group", and, any of this or that "nx' 1group" ) respective; 
At same time, "Fourth persons one of two composite concepts 四.A" (any of this or that "nx1-group", and, any of this or that "nx' 1-group"), whole in naturally corresponding "First person one composite concepts - " -" itself sync compound immortal-renew division and integration mutual-rebirth evolve pace, with "First person one composite concepts - " - $\left[N\left(\ldots \backslash 3 n x^{\prime} \backslash 2 n x^{\prime} \backslash 1 n x^{\prime} \backslash 1 n x \backslash 2 n x \backslash 3 n x \backslash \ldots, 0\right)\right]$ " consubstantiality relative, from "Second person one of two composite concepts 二.B" "0" sync compound rebirth-out, at category of "Second persons one of two composite concepts 二. $A^{\prime \prime}$ “. .. $\mid 3 n x^{\prime} \backslash 2 n x^{\prime} \backslash 1 n x^{\prime} \backslash 1 n x \backslash 2 n x \backslash 3 n x \backslash \ldots$ ", sync approximately hold balanced gather to compound (have life have death)-renew division and integration mutual-rebirth together evolve,

"Fourth persons one of two composite concepts 四.A" (any of this or that "..... $\mid 3 n x^{\prime} \backslash 2 n x^{\prime} \backslash 1 n x ' \backslash 1 n x \backslash 2 n x \backslash 3 n x$ (......", or any of this or that "nx1-group" ) ;

Above same one song played in different tone as below:

$<\ldots \backslash 3 n x^{\prime}-$ group $\backslash 2 n x^{\prime}$-group $\backslash 1 n x^{\prime}$-group $\backslash 1 n x-$ group $\backslash 2 n x$-group $\backslash 3 n x-g r o u p ~ \backslash \ldots>$

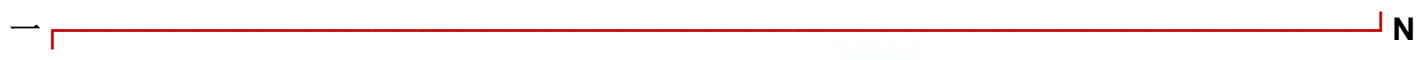

0

Above same one song played in different tone as below:

At same time sync vice versa.

Attached:

Mathematics "Positive and Negative Numbers Perfect System" of Science and "TAI CHI System" of The Book of Changes Similarly with above.

Principle model e.g.4. "TAI CHI System" of "The Book of Changes"

["Second person one of two composite concepts 二.A" ‘ "Third and Fourth persons three composite concepts" all "Yin, Yang, Yin-Yang together as one", whole corresponding, consubstantiality relative, from "Second person one of two composite concepts 二.B" "0-Yin Yi" sync compound rebirth-out, at category of "Second persons one of two composite concepts 二.A", sync approximately hold balanced compound (have life have death)-renew division and integration mutual-rebirth evolve respective and each other at same time, one-coincide sync consummate hold balanced compound immortal-renew division and integration mutual-rebirth evolve Yang Yi '... \3-Yin-nx' $\mid 2-Y i n n x^{\prime} \backslash 1$-Yin nx' \1-Yang-nx 2-Yang-nx \3-Yang-nx \..."'] ;

And.

"Second person one of two composite concepts 二.B" ‘ "Second persons one of two composite concepts 二.A" ("Consummate hold balanced "Third and Fourth persons three composite concepts" all "Yin, Yang, Yin-Yang together as one"), sync perfect evenly melt compound immortal-renew division and integration mutual-rebirth evolve Yin Yi" J \} respective;

Whole in naturally corresponding "First person one composite concepts -" "- Tai Chi" itself sync compound immortalrenew division and integration mutual-rebirth evolve pace,

With "First person one composite concepts -" "- Tai Chi 〔N ( '... I3-Yin-nx' \2-Yin nx' $\backslash 1$-Yin nx' \1-Yang-nx \2Yang-nx \3-Yang-nx \...' of Yang Yi、Yin Yi) ] " consubstantiality relative,

At exactly the same one position, sync compound immortal-renew division and integration mutual-rebirth (equal-amount exchange) evolve each other at same time, and at exactly the same one position, sync compound immortal-renew division and integration mutual-rebirth (equal-amount) together evolve,

"First person one composite concepts -" "- TAI CHI〔N ('... 13-Yin-nx' \2-Yin nx' \1-Yin nx' \1-Yang-nx \2-Yang$n x \backslash 3-Y a n g-n x \backslash . .$. ' of Yang Yi、Yin Yi ) ] ";

Above same one song played in different tone as below: 
Principle of 《The Book of Changes》〔 "Tai Chi" birth-rebirth "Dual trends the way"; "Dual trends the ways" birthrebirth "Four properties features (each one with $1 / 4$ amount of Tai-Chi)"; "Four properties features" birth-rebirth "Eight kinds of specific composition ways (each way all composite correspond have 'Dual trends the ways' and 'Four properties features', and each one with 1/8 amount of Tai-Chi)"; The same way keeps evenly divided until infinity; At same time sync on the contrary, Yin and Yang birth-rebirth each other and hugging together birth-rebirth "Tai-Chi" ] .

Above same one song played in different tone as below:

\section{YONG YI \\ TAI CHI $\longleftarrow$ YIN AND YANG BALL OF "TAI CHI"-N \\ YIN YI}

At same time sync vice versa.

\section{Principle model e.g.5. "Tao System" of "Tao Te Ching"}

\{"Second person one of two composite concepts 二.A" 〔 "Third and Fourth persons three composite concepts" all "Three or all", whole corresponding, consubstantiality relative, from "Second person one of two composite concepts 二.B" "Yin of Half Two" sync compound rebirth-out, at category of "Second persons one of two composite concepts 二.A", sync approximately hold balanced compound (have life have death)-renew division and integration mutual-rebirth evolve respective and each other at same time, one-coincide sync consummate hold balanced compound immortal-renew division and integration mutual-rebirth evolve Yang of Half Two"] ;

And.

"Second person one of two composite concepts 二.B" ( "Second persons one of two composite concepts 二.A" ("Consummate hold balanced "Third and Fourth persons three composite concepts" all "Three, or all"), sync perfect evenly melt compound immortal-renew division and integration mutual-rebirth evolve Yin of Half Two" ] \};

Whole in naturally corresponding "First person one composite concepts - "- Tao" itself sync compound immortal-renew division and integration mutual-rebirth evolve pace,

With "First person one composite concepts -" "- Tao [N (Yang of Half Two、Yin of Half Two) ]" consubstantiality relative,

At exactly the same one position, sync compound immortal-renew division and integration mutual-rebirth (equal-amount exchange) evolve each other at same time, and at exactly the same one position, sync compound immortal-renew division and integration mutual-rebirth (equal-amount) together evolve,

"First person one composite concepts -" "-〔N (Yang of Half Two、Yin of Half Two) ] of Tao";

Above same one song played in different tone as below:

Principle of 《Tao Te Ching》"One birth Two, two birth Three, Three birth All; At same time sync on the contrary, all the things leave from Yin and go to hug (and stay at) Yang, (Yin and Yang) aerated (each other) and merged to be one (Tao)".

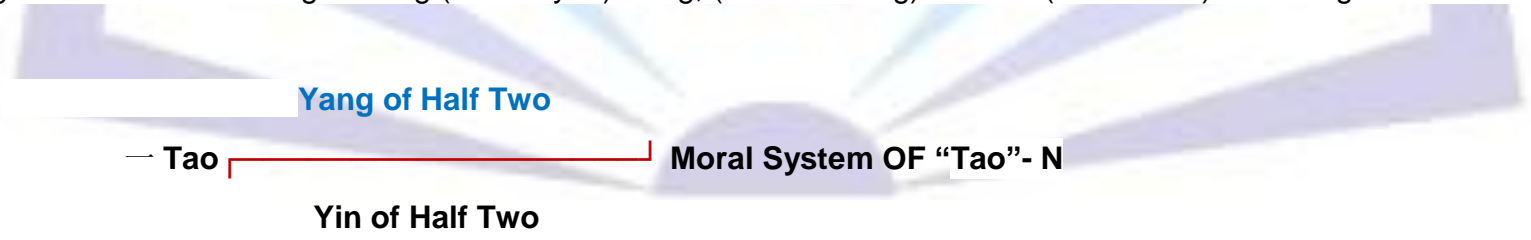

At same time sync vice versa.

\section{Principle model e.g.6.Trinity of Bible}

\{"Second person one of two composite concepts 二.A" ‘ "Third and Fourth persons three composite concepts" all "Son", whole corresponding, consubstantiality relative, from "Second person one of two composite concepts 二.B" "Holy Ghost" sync compound rebirth-out, at category of "Second persons one of two composite concepts 二.A", sync approximately hold balanced compound (have life have death)-renew division and integration mutual-rebirth evolve respective and each other at same time, one-coincide sync consummate hold mora balanced compound immortal-renew division and integration mutual-rebirth evolve Holy Body"] \};

And.

\{ "Second person one of two composite concepts 二.B" ‘ "Second persons one of two composite concepts 二.A" ("Consummate hold balanced "Third and Fourth persons three composite concepts" all "Son"), sync perfect evenly melt soul comprehension compound immortal-renew division and integration mutual-rebirth evolve Holy Ghost" ] \}; 
Whole in naturally corresponding "First person one composite concepts - " "- God" itself sync compound immortal-renew division and integration mutual-rebirth evolve pace,

With "First person one composite concepts - " - God〔 N ( Holy Body 、 Holy Ghost) 〕 of Trinity of Cross" consubstantiality relative,

At exactly the same one position, sync compound immortal-renew division and integration mutual-rebirth (equal-amount exchange) evolve each other at same time, and at exactly the same one position, sync compound immortal-renew division and integration mutual-rebirth (equal-amount) together evolve,

"First person one composite concepts -" "- God [N (Holy Body , Holy Ghost) ] of Trinity of Cross";

Above same one song played in different tone as below:

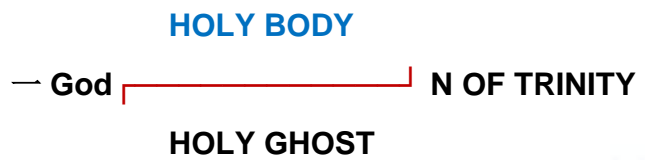

At same time sync vice versa.

\section{Principle model e.g.7.Three Kayas Buddha of Tantric}

\{"Second person one of two composite concepts 二.A" 〔 "Third and Fourth persons three composite concepts" all "(local)Three Kayas Buddha", whole corresponding, consubstantiality relative, from "Second person one of two composite concepts 二.B" "Female body Buddha" sync compound rebirth-out, at category of "Second persons one of two composite concepts 二.A", sync approximately hold balanced compound (have life have death)-renew division and integration mutual-rebirth evolve respective and each other at same time, one-coincide sync consummate hold balanced compound immortal-renew division and integration mutual-rebirth evolve Male body Buddha" ] ;

And.

"Second person one of two composite concepts 二.B" ( "Second persons one of two composite concepts 二.A" ("Consummate hold balanced "Third and Fourth persons three composite concepts" all "(local)Three Kayas Buddha"), sync perfect evenly melt compound immortal-renew division and integration mutual-rebirth evolve Female body Buddha" ]\};

Whole in naturally corresponding "First person one composite concepts -" "- Buddha" itself sync compound immortalrenew division and integration mutual-rebirth evolve pace,

With "First person one composite concepts - " - 〔N (Male body Buddha、Female body Buddha) ) of Three Kayas Buddha" consubstantiality relative,

At exactly the same one position, sync compound immortal-renew division and integration mutual-rebirth (equal-amount exchange) evolve each other at same time, and at exactly the same one position, sync compound immortal-renew division and integration mutual-rebirth (equal-amount) together evolve,

"First person one composite concepts -" "-〔N (Male body Buddha、Female body Buddha) $〕$ of Three Kayas Buddha";

Above same one song played in different tones as below:

Male body Buddha

- BUDDHA

N Of THREE KAYAS BUDDHA

Female body Buddha

At same time sync vice versa.

Etc.

Section 2. 《The principle of natural law》work with local

2.1. In "Third and Fourth persons three composite concepts",

Any of this or that " 1 ", is every other " 1 ",

Omni-dimensional inside and outside, 
Whole in naturally corresponding "First person one composite concepts -" "一" itself sync compound immortalrenew division and integration mutual-rebirth evolve pace,

With "First person one composite concepts -" “- $[N(1 \backslash n, 0)]$ " consubstantiality relative,

From "Second person one of two composite concepts 二.B" "0" sync compound rebirth-out,

At category of "Second persons one of two composite concepts 二.A", sync approximately compound (have life have death)-renew division and integration mutual-rebirth evolve into,

This or that "1";

At same time sync vice versa.

Attached:

Einstein's theory of relativity in category of "Third and Fourth persons three composite concepts", which mean mankind use different " 1 frequency type permutation combination orderly link" as measurable criteria will have different results.

2.2. In "Third and Fourth persons three composite concepts",

Any of this or that "1", and, every other "1",

Omni-dimensional inside and outside,

whole in naturally corresponding "First person one composite concepts - "一" itself sync compound immortal-renew division and integration mutual-rebirth evolve pace,

With "First person one composite concepts -" "- $[N(1 \backslash n, 0)]$ " consubstantiality relative,

From "Second person one of two composite concepts 二.B" "O" sync compound rebirth-out,

At category of "Second persons one of two composite concepts 二.A", sync approximately compound (have life have death)-renew division and integration mutual-rebirth evolve respective and each other.

2.3. "Fourth person one of two composite concepts 四.A" this or that "nx1-group" series,

And,

"Fourth person one of two composite concepts 四.B" this or that " $n x^{\prime} 1$ " series,

Omni-dimensional inside and outside,

Whole in naturally corresponding "First person one composite concepts -" "一" itself sync compound immortal-renew division and integration mutual-rebirth evolve pace,

With ["First person one composite concepts - " - $[N(1 \backslash n, 0)]$ " and "Third person one composite concepts 三” this or that "1 $\left(\mathbf{n}\left(\mathrm{n} \times 1\right.\right.$-group series、 $\mathbf{n} \mathbf{x}^{\prime} 1$ series $\left.\left.)\right)\right\}$ respective consubstantiality relative,

From "Second person one of two composite concepts 二.B" "0" sync compound rebirth-out,

At category of "Second persons one of two composite concepts 二.A", approximately same one position, sync compound (have life have death)-renew division and integration mutual-rebirth (approximately equal amount exchange) evolve each other at same time, approximately same one position, sync gather to compound (have life have death)-renew division and integration mutual-rebirth (approximately equal amount) together evolve into,

"Third person one composite concepts 三” this or that "1 $(n(n \times 1$-group series $\mathbf{n x}$ ' 1 series $))$;

At same time sync vice versa.

Attached:

"Particle Spin Local-link" of Science, "Local-Trinity" of religion Bible, "Yin and Yang birth-rebirth each other and hugging together (birth-rebirth local-one)" of China "The Book of Changes", "Leave from Yin and go to hug Yang, (Yin and Yang) aerated (each other) and merged (to be local-one)" of China "Tao Te Ching" , "Three (local) Kayas Buddha" of TibetanTantric etc, all are same one song played in different tone as above 2.3.

Among them:

Basis on the "Fourth persons one of two composite concepts 四.A" this or that "nx1-group", and, "Fourth person one of two composite concepts 四. B" this or that " $n x^{\prime}$ 1", composite correspond each other:

"Fourth person one of two composite concepts 四.B" this or that " $n x^{\prime}$ 1" is "Fourth persons one of two composite concepts 四.A" this or that "nx1-group" approximately hold balanced every "1", approximately evenly melt "Commonness memory space and time of $n x^{\prime} 1$-link"; This or that " $n x^{\prime}$ 1-link structure" is less than "Fourth persons one of two composite concepts 四. A" in this or that "nx1-group" any "1" its "structure", but it "volume amount" big then than "Fourth persons one of two composite concepts 四.A" in this or that "nx1-group" any "1" its "volume amount". And it is "Fourth persons one of 
two composite concepts 四.A" in this or that "nx1-group" any "1" living space; It tend to and show "Second person one of two composite concepts 二.B" "Perfect evenly melt commonness memory space and time of zero distance" standpoint and attributes, so physical needs to improves as "Common particle".

"Fourth persons one of two composite concepts 四.A" this or that "nx1-group" with its own approximately hold balanced every " 1 ", tend to and show "Second persons one of two composite concepts 二.A" "Consummate hold -balanced together booming order", so physical needs to improves here every "1" as "Personalized particle".

"Common particle" and "Personalized particle" coexist in (large-small)-dimension structure of universe.

If scientist use "Fourth persons one of two composite concepts 四.B" "Common particle" as explore the universe's energy power, "Common particle" will come with "about evenly powerful volume amount" in short distance fast come to its scope "Fourth persons one of two composite concepts 四.A" every every " 1 Personalized particle". And more close "zero Common Primitives", It more powerful and more fast shorten the distance. So "Common particle" is the "Time tunnel" that scientist look for.

If scientist use "Common particle" as "explosive" as nuclear weapons, it destroys people's life and natural same powerful and fast as above.

Combine with next 2.4. the scientist should be right way stop use Collider's opposition and confrontation to find out deep structural. Because if people exploded "Common particle" level, Human world will collapse and instantly disappear. The use of chemical seek fusion structural should be more safe.

Also in physics, "Fourth persons one of two composite concepts 四.A" "Group local time and space particles approximately hold-balanced together booming order of Tension-equation nx-group" , Its amount composite correspond approximatelyequal to, "Fourth person one of two composite concepts 四.B" "nx' time and space Gravitational Particle-Field amount". So the scientist could from this way find out amount of gravitational volume amount.

For e.g. sociology combine with "Theorem Three", in "Third person one composite concepts 三”, if many "1 (n ( nx1-group series $\mathbf{n} \mathbf{x}^{\prime} 1$ series) I person (or party, or country, or etc.)" try to find out commonness together, they could keep respective "Third person one composite concepts 三” standpoint and attributes at same time, change respective and together approximately balance (Tension) become "Fourth person one of two composite concepts 四.A" this or that " $n \times 1$ group" standpoint and attributes; Then they could find out their together have in "Fourth person one of two composite concepts 四.B" this or that “ (approximately evenly melt commonness memory soul (space and time of Gravitational Field) ) $n x^{\prime}$ 1"; And they could find out they are together have in "Third person one composite concepts 三” "1 ( $n$ ( $n \times 1-g r o u p$ series、 $\mathbf{n} \mathbf{x}^{\prime} 1$ series) ) achievement (could be party, or country, or Earth human community, or etc)"; At same time sync vice versa (for e.g. academic research achievement).

2.4. In "Third and Fourth persons three composite concepts",

Any Omni-dimensional direction inside and outside to antithesis and confrontation "1",

Whole in naturally corresponding "First person one composite concept S -" "一" itself sync compound immortal-renew division and integration mutual-rebirth evolve pace,

With "First person one composite concepts -" “- $[N(1 \backslash n, 0)]$ " consubstantiality relative,

From "Second person one of two composite concepts 二.B" "0" ablation own personality at the same time sync compound rebirth-out,

At category of "Second persons one of two composite concepts 二.A", back and close to before lifetime position, from near and far sync compound (have life have death)-renew division and integration mutual-rebirth evolve into,

"Third person one composite concepts" new part of all "1 ( $n(n \times 1$-group series、 $\mathbf{n x}$ ' 1 series ) ) ;

At same time sync vice versa.

Attached:

For e.g. Every generation of virus transform back to the world, it will be more strong against human life, living, survival and development.

Combine "Fourth person one of two composite concepts 四.B" and "Theorem Three", "Third person one composite concepts 三” any this or that "1 ( $n$ ( nx1-group series、 nx' 1 series) ) human" have darth, his or her "Fourth person one of two composite concepts 四.B" "nx' 1-('Time and space' of 'commonness memory soul')" not die at same time, and if people not at lifetime mastery their own and others experiences and knowledge, not understand all the "Presence Ways and Presence Relationships", That is not understand 《The principle of natural law》, then people will no key to solve their from lifetime got these memory problems, all the memory in soul will keep antithesis and confrontation, soul's own "time and space" of survive will be more and more crowded, feeling just like can not breathe, until "time and space of soul" again broken-die like a bubble. Because "Fourth person one of two composite concepts 四.B" "nx' 1-('Time and space' of 'commonness memory soul')" also having "Third person one composite concepts 三” "1 $(n$ ( $n \times 1$-group series、 nx' 1 series) ) Soul" standpoint and attributes, so soul's soul will be keep blowout-die, until to its memory all have conversion. This process is very painful, all the major religions describes as hellish living. 
In "Third and Fourth persons three composite concepts", any "1-soul" with animal's sperm and eggs combined again called "reincarnation", reincarnation's soul its new memory day by day build up and naturally push old memory savings to the depths, so human limitation from focus on the new life and old memory naturally degenerate, the people follow that time growth more, the old memory push away more, until the person lost them in deep. Science has been limited by horizons, it need time mastery their own and others experiences and knowledge to got ultimate truth. During this time, arrogant science exclusion of ancient human civilization and culture, self-wrapped cocoon.

So human need learning what is life, also need learning what is death. Knowing mankind where come from, where are mankind going, what is mankind life meaning. Human don't understand life and death, human beings on their own irresponsible, human's life suffering and after death suffering, endless.

2.5.1. In "Third and Fourth persons three composite concepts",

Not inclusive any two "1",

Omni-dimensional inside and outside,

Whole in naturally corresponding "First person one composite concept s -" "一" itself sync compound immortal-renew division and integration mutual-rebirth evolve pace,

With "First person one composite concepts -" “- $[N(1 \backslash n, 0)]$ " consubstantiality relative,

From "Second person one of two composite concepts 二.B" "O" sync compound rebirth-out,

At category of "Second persons one of two composite concepts 二.A", sync approximately compound (have life have death)-renew division and integration mutual-rebirth together evolve into,

This or that "1-linear";

At same time sync vice versa.

Attached:

2.5.2. In "Third and Fourth persons three composite concepts",

Any this "1" inclusive that "1" become,

That "1" with many of "1" that come from this "1 ( $\mathrm{n}$ ( $\mathrm{nx} 1$-group series、 $\mathbf{n x}$ ' 1 series ) ) ,

Omni-dimensional inside and outside,

Whole in naturally corresponding "First person one composite concept s - "一" itself sync compound immortal-renew division and integration mutual-rebirth evolve pace,

With "First person one composite concepts - " $[N(1 \backslash n, 0)]$ " consubstantiality relative,

From "Second person one of two composite concepts 二.B" "0" sync compound rebirth-out,

At category of "Second persons one of two composite concepts 二.A", sync approximately compound (have life have death)-renew division and integration mutual-rebirth together evolve into,

This or that "1-nonlinear";

At same time sync vice versa.

Attached:

Basis on the above, people find out more "1" that come from this "1 ( $\mathrm{n}$ ( $\mathrm{nx}$ 1-group series、 $\mathbf{n x}$ ' 1 series) ) with That "1" relationship, there will be more rich connotation and denotation about this or that "1-nonlinear".

2.5.3. In "Third and Fourth persons three composite concepts",

Any mutually inclusive two " 1 ",

Departure from their respective standpoint and attributes,

Omni-dimensional inside and outside,

Whole in naturally corresponding "First person one composite concept s - "一" itself sync compound immortal-renew division and integration mutual-rebirth evolve pace,

With "First person one composite concepts -" "- $[N(1 \backslash n, 0)]$ " consubstantiality relative,

From "Second person one of two composite concepts 二.B" "0" sync compound rebirth-out,

At category of "Second persons one of two composite concepts 二.A", sync approximately compound (have life have death)-renew division and integration mutual-rebirth together evolve into,

Any of this or that "1-common"; 
At same time sync vice versa.

Attached:

This also mean in "Third and Fourth persons three composite concepts", when this and that " 1 " come to each other standpoint and attributes, they are become one "1-common thing" with different name and different way to clarify. Otherwise will be same as example "Solid ball", people will each stick to their own limited area, disagree and no-inclusive other side.

2.6. In "Fourth persons one of two composite concepts 四.A",

Any of this or that "nx1-group series",

And,

Any of this or that " $\mathrm{nx}$ ' 1-group series",

Omni-dimensional inside and outside,

Whole in naturally corresponding "First person one composite concept s - "一" itself sync compound immortal-renew division and integration mutual-rebirth evolve pace,

With ["First person one composite concepts -" "-〔N ( ... $\left.\left.3 n x^{\prime} \backslash 2 n x^{\prime} \backslash 1 n x^{\prime} \backslash 1 n x \backslash 2 n x \backslash 3 n x \backslash \ldots, 0\right)\right]$ " and "Fourth persons one of two composite concepts 四.A" this or that (any of this or that “..... $\mid 3 n x^{\prime} \backslash 2 n x^{\prime} \backslash 1 n x^{\prime} \backslash 1 n x \backslash 2 n x \backslash 3 n x$ \......, or any of this or that "nx1-group") \} consubstantiality relative,

From "Second person one of two composite concepts 二.B" "O" sync compound rebirth-out,

At category of "Second persons one of two composite concepts 二.A “... $3 n x^{\prime} \backslash 2 n x^{\prime} \backslash 1 n x^{\prime} \backslash 1 n x \backslash 2 n x \backslash 3 n x \backslash \ldots$ ”, sync approximately hold balanced and compound (have life have death)-renew division and integration mutual-rebirth together evolve into,

Any of this or that (any of this or that "..... $13 n x^{\prime} \backslash 2 n x^{\prime} \backslash 1 n x^{\prime} \backslash 1 n x \backslash 2 n x \backslash 3 n x \backslash \ldots \ldots$., or any of this or that "nx1-group");

At same time sync vice versa.

2.7. "Third and Fourth persons three composite concepts" respective,

Omni-dimensional inside and outside,

Whole corresponding, consubstantiality relative,

except their own every others,

and (including themselves all "Third and Fourth persons three composite concepts" together, participation division and integration, together have in common of "First and Second persons three composite concepts" respective),

all have their own different functions of "level, angle, position, interface".

"Third and Fourth persons three composite concepts" respective is each other deep composition;

"Second person one of two composite concepts" are "Third and Fourth persons three composite concepts" together have in common "Consummate hold balanced" and "Perfect evenly melt" of two half body;

"First person one composite concepts" is "Third and Fourth persons three composite concepts" together have in common completely one body;

At same time sync on the contrary, "Third and Fourth persons three composite concepts" respective, all is part of "First and Second persons three composite concepts";

"Third and Fourth persons three composite concepts" all "1" face "First and Second persons three composite concepts" respective, all is equal footing but function different.

\section{Section 3. 《Natural Human Development Brief History》}

Combine with Theorem One Section 1 "Third person one composite concepts". in "Third and Fourth persons three composite concepts",

When "1〔n (nx1-group、 nx'1) 〕 of triple approximately compound frequency type permutation-combination orderly spin link" series not show up yet, we name them dark matter, dark energy, dark bio etc.

When "1〔 $n$ (nx1-group、 $\left.n x^{\prime} 1\right)$ of triple approximately compound frequency type permutation-combination orderly spin link" of series show up but chaos and unclear, we name them energy.

When "1〔 $n$ (nx1-group、 $\left.\left.n x^{\prime} 1\right)\right]$ of triple approximately compound frequency type permutation-combination orderly spin link" series show up clear and stable, we name them substance or .

When "1[n (nx1-group、 $\left.\left.n x^{\prime} 1\right)\right]$ of triple approximately compound frequency type permutation-combination orderly spin link" series start know "between me and non-itself" are different, "between me and It or she or he" are different. Including 
microbiological and biological viruses, we name them Biological and Animals. The reaction and reflection build the animal's Brain organization, which are structure of rational consciousness.

Human body of " $1\left[n\left(n \times 1-g r o u p 、 n x^{\prime} 1\right)\right)$ of triple approximately compound frequency type permutation-combination orderly spin link" much more clear and stretch than any of other animals. So our Human all have a physiological condition to understand why things are this or that way; what are relationships between them. This mean all human could be figuring out "human where come from, human where to go, what are human life meaning". After human got the real answer. Human will realize that things and the real truth, could from each of human position, by different names and different ways build up. Basis on the understanding balance and respect each other, humans will naturally move towards settlement and consensus, accordingly live in the peace.

When "1〔 $n$ (nx1-group、 $\left.\left.n x^{\prime} 1\right)\right]$ of triple approximately compound frequency type permutation-combination orderly spin link" series approaching biological, we name them plants, alga, etc.

Etc.

Early humans body of " $1[n(n \times 1-g r o u p 、 n x ' 1)]$ of triple approximately compound frequency type permutationcombination orderly spin link" much more chaos than lately human, so that is why early humans could feel and see some type of energy (It including "human common memory of soul" etc.) ; At the same time energy vice versa renew-rebirth human's. Mankind into "witchcraft era of many human groups civilization cultures".

When the human body of " $1\left[n\left(n \times 1\right.\right.$-group、 $\left.n x^{\prime} 1\right) 〕$ of triple approximately compound frequency type permutationcombination orderly spin link" complex develop much more clear, so humans also could clearly feel and see the structural substance then before; At the same time substance vice versa renew-rebirth human's body. Flow the time, Human also day by day lost function to feel and see the energy. During this time some humans (very few) master their own and others experience and knowledge got the great whole truth. That is "A monotheistic religion" and "Philosophy unified theory" start it. And it led the human build up "The civilization and culture era of many state social unification".

When the human body of " $1\left[n\left(n \times 1-g r o u p 、 n x^{\prime} 1\right)\right]$ of triple approximately compound frequency type permutationcombination orderly spin link" Clearly and deeply complex development. Human cognitive certification "(large-small)-scale structure of universe"; At the same time (large-small)-scale structure of universe vice versa renew-rebirth human's body. This lead human start builds up "Scientific (civilization and culture) era of globe human social unification".

Etc.

Theorem Three: "Basis's own department with its naturally composite correspond standpoint and attributes" and "Persons-Concepts、Position、Trend, these three key elements, jointly determine "Six composite concepts of Quadrity", whole corresponding, consubstantiality relative, at same time sync keep (and 、or) conversion respective self or each other, naturally composite correspond standpoint and attributes" --Complete Relativistic and Complete Dialectics

\section{Section 1. Basis's own department with its natural composite correspond standpoint and attributes}

Take Eastern Western all had outstanding remarkable "Trinity" as example, combine with Theorem one and Theorem Two:

1.1. First-Person: "Basis's own department" 《The principle of natural law》"Complete-Universe", it has same one song played in different tones as, (Bible "God" and "Trinity" and "cross"; Buddhist scriptures "Buddha", Tantric "Three Kayas Buddha"; Western "Noumenon"; The Book of Changes "Yin Yang Ball”; Quran "Allah"; Tao Te Ching "Tao" etc.) composite correspond show and unfold:

Second-Person: From human function formatting 《The principle of natural law》(Structured) "First person one composite concepts - " - $[N(1 \backslash n, 0)]$ of triple completely compound spin system", it has same one song played in different tones as ‘ Bible "God of moral tangible system", "Trinity of Father, Son, Holy Spirit (and Holy Body)", "One vertical of Cross"; Buddhist scriptures "Buddha-Dharma"; Tantric "Male Buddha of Three Kayas completely Buddha"; Western "Being"; The Book of Changes "Tai Chi of Yin Yang Ball", "Yang of Yin Yang Ball"; Quran "(Man white dress to show) Moral tangible system of Allah"; Tao Te Ching "Tao Law" etc. J (which is Theorem one.1.).

And,

Third-Person: Not from human function formatting 《The principle of natural law》 "No human language could described Complete-Universe", it has same one song played in different tones as (Quran "(Woman black dress to show) Allah invisible" ; Bible "God invisible"; Buddhist scriptures "Nirvana", Tantric "Female Buddha of Three Kayas completely Buddha"; Western "Nothingness"; The Book of Changes "Wu Chi", "Yin of Yin Yang Ball"; Tao Te Ching "Nothing" etc. J (which is Theorem one.2.).

Above three persons composite correspond establish Trinity. Among them "Second person" and "Third-Person" both are "First-Person" its own showed in different ways. These three persons are each other and as one.

Below anew separate to show Quran and Bible to help Islamists and Christian understand each other, respect each other. 
Quran

First-Person: Allah's own department composite correspond to show:

Second-Person: Perfect moral structure system of Allah (Man white dress to show moral tangible Allah).

Third-Person: No human language could describe Allah (Woman black dress and covering to show Allah invisible).

Above three persons composite correspond establish Trinity. These three persons are each other. Three persons is one Allah.

Bible

First-Person: God and cross's own department composite correspond to show:

Second-Person: Perfect moral structure spin system of God (Trinity of "Father, Holy Ghost and Holy Body, Son, three in one", One of the components of the Cross).

Third-Person: No human language could describe God (One of another component of the Cross).

Above three persons composite correspond establish Trinity. These three persons are each other. Three persons is one God.

1.2. So Quran and Bible both had Basis-Trinity's own department. Basis on the above, in view of most consciousness build by tangible Limitations of part, once people mastery their own and others experiences and knowledge:

First-Person: Human will be naturally composite correspond:

Second-Person: Life learn above "1.1. Second-Person", understanding and balance together, tend to moral tangible Trinity of complete "Own-God";

Third-Person: Death learn above "1.1. Third-Person", not hold body's respectively consciousness, return to invisible of no limitations of perfect "Own-God".

Above three persons composite correspond establish Trinity. But this Trinity come from 1.1.

Combine with Theorem Two 2.4.

"Death of antithesis and confrontation", and, "Death learn as '1.1. Third-Person', not hold body's respectively consciousness, return to invisible of no limitations of perfect 'Own-God"', They are not the same death. The former in "1.1. Second-Person" its Trinity of structure and very painful transformation transmigration; The latter no longer having consciousness the distinguish, and no longer transmigration.

Etc.

Below all Trinity based on the above "1.1. Trinity".

Section 2. "Six composite concepts of Quadrity" respective 's own department with their naturally composite correspond standpoint and attributes

Take Eastern Western all had outstanding remarkable "Trinity" as example, and combine with "Theorem one Section .1." and Theorem Two:

\section{First person one composite concepts -}

1.1. First-Person: "- $[N(1 \backslash n, 0)]$ of triple completely compound spin system"; it has same one song played in different tones as: 〔Bible "God of moral tangible system”, "Trinity of Father, Son, Holy Spirit (and Holy Body)", "Cross"; Buddhist scriptures "Buddha-Dharma"; Tantric "Three Kayas Buddha"; Western "Being"; The Book of Changes "Yin Yang Ball” ; Quran "(Man white dress to show) Moral tangible system of Allah"; Tao Te Ching "Tao Law"; Science "Wave particle complete duality", Science "Particle complete Spin" etc. ] every one's own department, with their respective:

Second person: "Divide-trends";

And,

Third person: "Integration-trends";

Above three persons composite correspond establish Trinity. These three persons are each other.

1.2. First-Person: "First person one composite concepts - "- $[N(1 \backslash n, 0)]$ of triple completely compound spin system"; it has same one song played in different tones as: ¿ Bible "God of moral tangible system--Trinity of 'Father, Son, Holy Spirit (and Holy Body)", "Cross"; Tantric "Three Kayas Buddha”; The Book of Changes "Tai Chi System", "Yin Yang Ball"; Tao Te Ching "Tao"; Science "Wave particle complete duality", Science "Particle complete Spin" etc. J every one's own department, with their respective two perfect half equal-amount of body:

Second person: "Second person one of two composite concepts 二.A" "1 \n"; it has same one song played in different tones as: 〔Bible "Holy Body", "One of component of the Cross"; Tantric "Male Body Buddha"; The Book of Changes "Yang Yi", "Yang of Yin Yang Ball"; Science "All local time and space particles consummate hold -balanced together booming order of Tension-equation $1 \backslash n$ " (Its amount composite correspond conservation-equal to "Second person one of 
two composite concepts 二.B" "Zero time and space Gravitational Primitives-Field amount"). "Wave-Particle Consummate Hold Balanced Duality" etc. ] .

and,

Third-Person: "Second person one of two composite concepts 二.B" "0"; it has same one song played in different tones as: 〔Bible "Holy Ghost", "One of another component of the Cross"; Tantric "Female Body Buddha"; The Book of Changes "Yin Yi", "Yin of Yin Yang Ball"; Science "Zero time and space Gravitational-Primitives-Field (Its amount composite correspond conservation-equal to "Second person one of two composite concepts 二.A" "All local time and space particles consummate hold -balanced together booming order of Tension-equation $1 \backslash \mathrm{n}$ amount) etc. ] .

Above three persons composite correspond establish Trinity. These three persons are not each other.

1.3. First person: "First person one composite concepts - "- $[N(1 \backslash n, 0) 〕$ of triple completely compound spin system"; it has same one song played in different tones as: 〔Bible "God of moral tangible system--Trinity of Father, Son, Holy Spirit (and Holy Body)", "Cross"; Tantric "Three Kayas Buddha”; The Book of Changes "Tai Chi System", "Yin Yang Ball”; Tao Te Ching "Tao Law" etc. ] every one's own department, with their respective two "half equal amount" body:

Second person: "Second person one of two composite concepts 二.A" "1 $\backslash n$ "; it has same one song played in different tones as: 〔Bible "Holy Body", "One of component of the Cross"; Tantric "Male Body Buddha"; The Book of Changes "Yang Yi", "Yang of Yin Yang Ball"; Science "All local time and space particles consummate hold -balanced together booming order of Tension-equation $1 \backslash \mathrm{n}$ " (Its amount composite correspond conservation-equal to "Second person one of two composite concepts 二.B" "Zero time and space Gravitational Primitives-Field amount"). "Wave-Particle Consummate Hold Balanced Duality" etc. ] \}, and, \{ "Second person one of two composite concepts 二.B" "0"; it has same one song played in different tones such as: 〔Bible "Holy Ghost", "One of another component of the Cross"; Tantric "Female Body Buddha"; The Book of Changes "Yin Yi", "Yin of Yin Yang Ball"; Science "Zero time and space Gravitational-PrimitivesField (Its amount composite correspond conservation-equal to "Second person one of two composite concepts 二.A" "All local time and space particles consummate hold -balanced together booming order of Tension-equation $1 \backslash \mathrm{n}$ amount) etc. ] \}.

And,

Third-Person: "Third and Fourth persons three composite concepts" all "1"; it has same one song played in different tones as: (Bible all "son", "On the Cross Christ"; Tantric "Local Three Kayas Buddha"; The Book of Changes "Yin and Yang birth each other and hugging together", "In the Yin Yang Ball the dot"; Science "Local wave-particle duality", Science "Local particle Spin"; etc.).

Above three persons composite correspond establish Trinity. These three persons are not each other.

Etc.

\section{Second person one of two composite concepts 二.A}

First person: "1\n"; it has same one song played in different tones as: 〔Bible "Holy Body", "One of component of the Cross"; Tantric "Male Body Buddha"; The Book of Changes "Yang Yi", "Yang Yi of Yin Yang Ball"; Science "All local time and space particles consummate hold-balanced together booming order of Tension-equation 1 $\backslash n$ " (Its amount composite correspond conservation-equal to "Second person one of two composite concepts 二.B" "Zero time and space Gravitational Primitives-Field amount”). "Wave-Particle Consummate Hold Balanced Duality" etc. J every one's own department, with their respective:

Second person: "Divide-trends";

And,

Third person: "Integration-trends";

Above three persons composite correspond establish Trinity. These three persons are each other.

Etc.

\section{Second person one of two composite concepts 二.B}

First person: "0"; it has same one song played in different tones as: 〔Bible "Holy Ghost", "One of another component of the Cross"; Tantric "Female Body Buddha"; The Book of Changes "Yin Yi", "Yin Yi of Yin Yang Ball"; Science "Zero time and space Gravitational-Primitives-Field (Its amount composite correspond conservation-equal to "Second person one of two composite concepts 二.A" "All local time and space particles consummate hold -balanced together booming order of Tension-equation $1 \backslash \mathrm{n}$ amount) etc. J every one's own department, with their respective:

Second person: "Divide-trends";

And,

Third person: "Integration-trends"; 
Above three persons composite correspond establish Trinity. This three persons are each other.

Etc.

Third person one composite concepts 三

1.1. First person: "Third and Fourth persons three composite concepts" all "1"; it has same one song played in different tones such as: (Bible all "son", "On the Cross Christ"; Tantric "Local Three Kayas Buddha"; The Book of Changes "Yin and Yang birth each other and Hugging together", "In the Yin Yang Ball the dot"; Science "Local wave-particle duality", Science "Local particle Spin" etc.) every one's own department, with their respective:

Second person: "Divide-trends";

And,

Third person: "Integration-trends";

Above three persons composite correspond establish Trinity. These three persons are each other.

1.2. First person: "Third and Fourth persons three composite concepts" all " 1 [ $n$ ( $n \times 1$-group series、 $n x ' 1$ series) )" respective; they all have same one song played in different tones as: (Bible all "son", "On the Cross Christ"; Tantric "Local Three Kayas Buddha"; The Book of Changes "Yin and Yang birth each other and Hugging together", "In the Yin Yang Ball a dot"; Science "Local wave-particle duality", Science "Local particle Spin" etc.) every one's own department, with their two approximately half equal-amount of body:

Second person: "Fourth person one of two composite concepts 四.A" "nx-group"; it has same one song played in different tones as: ‘ Bible "Body of moral balance", "One of component of the Cross"; Tantric "Male Body Buddha"; The Book of Changes "Yang", "Yang of Yin Yang Ball"; Science "Group local time and space particles approximately hold -balanced together booming order of Tension-equation nx-group" (Its amount composite correspond approximately-equal to "Fourth person one of two composite concepts 四.B" "nx' time and space Gravitational Particle-Field amount"). "Wave-Particle Approximately Hold Balanced Duality" etc. ]

and,

Third-Person: "Fourth person one of two composite concepts 四.B" " $\mathrm{nx}$ ' "; it has same one song played in different tones as: ¿ Bible "Soul comprehension", "One of another component of the Cross"; Tantric "Female Body Buddha"; The Book of Changes "Yin", "Yin of Yin Yang Ball"; Science "nx' time and space Gravitational Particle-Field amount" (Its amount composite correspond approximately-equal to "Fourth person one of two composite concepts 四.A" "Group local time and space particles approximately hold-balanced together booming order of Tension-equation nx-group amount"). "WaveParticle Approximately Evenly Melt Duality" etc. ] .

Above three persons composite correspond establish Trinity. These three persons are not each other.

Etc.

\section{Fourth person one of two composite concepts 四.A}

First person: "nx-group", it has same one song played in different tones as: 〔Bible "Body of moral balance", "One of component of the Cross"; Tantric "Male Body Buddha"; The Book of Changes "Yang", "Yang of Yin Yang Ball"; Science "Group local time and space particles approximately hold -balanced together booming order of Tension-equation nxgroup" (Its amount composite correspond approximately-equal to "Fourth person one of two composite concepts 四.B" “nx' time and space Gravitational Particle-Field amount"). "Wave-Particle Approximately Hold Balanced Duality" etc. J every one's own department, with their respective:

Second person: "Divide-trends";

And,

Third person: "Integration-trends";

Above three persons composite correspond establish Trinity. These three persons are each other.

Etc.

\section{Fourth person one of two composite concepts 四.B}

First person: "nx' ", it has same one song played in different tones as: (Bible "Soul comprehension", "One of another component of the Cross"; Tantric "Female Body Buddha"; The Book of Changes "Yin", "Yin of Yin Yang Ball"; Science "nx' time and space Gravitational Particle-Field amount" (Its amount composite correspond approximately-equal to "Fourth person one of two composite concepts 四.A" "Group local time and space particles approximately hold-balanced together booming order of Tension-equation nx-group amount"). "Wave-Particle Approximately Evenly Melt Duality" etc. J every one's own department, with their respective:

Second person: "Divide-trends";

And, 
Third person: "Integration-trends";

Above three persons composite correspond establish Trinity. These three persons are each other.

Etc.

\section{Section 3.}

1.1. Combine with Theorem Two 1.

Second person: "Second person one of two composite concepts 二.A" "1 $\backslash n$ "; it has same one song played in different tones such as: 〔 Bible "Holy Body", "One of component of the Cross"; Tantric "Male Body Buddha"; The Book of Changes "Yang Yi", "Yang of Yin Yang Ball"; Science "All local time and space particles consummate hold-balanced together booming order of Tension-equation $1 \backslash \mathrm{n}$ " (Its amount composite correspond conservation-equal to "Second person one of two composite concepts 二.B" "Zero time and space Gravitational Primitives-Field amount"). "Wave-Particle Consummate Hold Balanced Duality" etc. ] \} respective, in the disappearance of their own presence way at same time, transform their own converted into "Second person one of two composite concepts 二.B" "0"; it has same one song played in different tones such as: 〔 Bible "Holy Ghost", "One of another component of the Cross"; Tantric "Female Body Buddha"; The Book of Changes "Yin Yi", "Yin of Yin Yang Ball"; Science "Zero time and space Gravitational-Primitives-Field (Its amount composite correspond conservation-equal to "Second person one of two composite concepts 二.A" "All local time and space particles consummate hold-balanced together booming order of Tension-equation $1 \backslash$ n amount) etc. J \}; At same time sync vice versa.

"Fourth person two composite concepts 四.A and 四.B" similarly as above.

\subsection{Combine with Theorem Two 2.4 .}

In "Third and Fourth persons three composite concepts 三 and 四.A and 四.B" any one, Omni-dimensional direction inside and outside to antithesis and confrontation, in the disappearance of their own presence way at same time, transform their own converted into new part of “Third and Fourth persons three composite concepts 三 and 四.A and 四.B" all others.

\section{Section 4.}

"Third and Fourth persons three composite concepts 三 and 四.A and 四.B" respective keep their own standpoint and attributes at same time, sync conversion into each other standpoint and attributes.

Among them, "Fourth person one of two composite concepts 四.B" change its "Frequency type permutation-combination orderly nx1-group" into "1〔 $n$ ( $n \times 1$-group series、 $n x$ '1 series) 〕 of triple frequency type permutation-combination orderly spin link"; At same time sync vice versa.

\section{Section 5.}

Combine with example "The complete universe is a solid ball", Humans different civilizations and cultures reach to each other standpoint and attributes, all keep their own standpoint and attributes at same time, sync conversion into each other standpoint and attributes, all become the same one song played in different tones (as each other or each own). Otherwise people will each stick to their own limited area, disagree and no-inclusive other side.

Etc.

So as above, "Basis's own department with its naturally composite correspond standpoint and attributes" and "Personsconcepts, Position, Trend, these three key elements, jointly determine "Six composite concepts of Quadrity", whole corresponding, consubstantiality relative, at same time sync keep (and or) conversion respective self or each other, naturally composite correspond standpoint and attributes"--Complete Relativistic and Complete Dialectics.

Well done.

\section{Summary}

《The principle of natural law》 come up with "Three basis theorem", they are supplementary to each other, complement each other, play a part together as:

Theorem one: "Cornerstone of principle--Six composite concepts of Quadrity" and "Complete Universe's own department of Trinity".

Theorem Two: "Basis on 'synchronous multi-D mutual-birth generate frequency, synchronous multi-D mutual-rebirth generate frequency cycle', Principle show and unfold in six composite concepts Quadrity, sync compound (immortal, immortal with have-life-have-death, have-life-have-death)-renew division and integration mutual-rebirth evolve each own and mutual presence ways and presence relationships and build in Complete mathematical modeling".

Theorem Three: "Basis's own department with its composite-natural-correspond standpoint and attributes" and "PersonsConcepts、Position、Trend, these three key elements, jointly determine "Six composite concepts of Quadrity", whole corresponding, consubstantiality relative, at same time sync keep (and or) conversion respective self or each other, naturally composite correspond standpoint and attributes"--Complete Relativistic and Complete Dialectics. 
All human cognitive and certification all could find from this "Three basis theorem". All the "Presence Ways and Presence Relationships" could use this "Three basis theorem" clarify.

So "Three basis theorem" :

In accordance with "Cornerstone of principle--Six composite concepts of Quadrity" and "Complete Universe's own department of Trinity" classify rejoin all human cognition certification.

Translation one same thing had different names and different ways into each other.

Help all civilizations, cultures, disciplines each other strengths and weaknesses complementary.

Help establish and improve all civilizations, cultures, disciplines respective missing.

Fully elucidated that human how cognition certification universe and life, Universe and life will be how open (information) to human; Human have how many ways to understanding universe and life, Human will have how many ways to prove it (including metaphysical); Human understanding how much universe and life, Universe and life will be how much open to the human.

Among "Three basis theorem":

1.1. "First and Second persons three composite concepts" respective and each other, all is "Presence Ways and Presence Relationships" of "sync compound immortal-renew division and integration mutual-rebirth evolve".

"Third and Fourth persons three composite concepts" respective and each other, all is "Presence Ways and Presence Relationships" of "sync compound (have life have death)-renew division and integration mutual-rebirth evolve".

("First and Second persons three composite concepts" respective, with, "Third and Fourth persons three composite concepts" respective), all is "Presence Ways and Presence Relationships" of "sync compound 〔immortal、immortal and (have life have death), have life have death ] -renew division and integration mutual-rebirth evolve".

1.2. "First person one composite concepts" is "Second and Third and Fourth persons five composite concepts" respective own and everyone together, have in common one complete body; At same time sync on the contrary, "Second and Third and Fourth persons five composite concepts" are "First person one composite concepts" its own part of composition.

"First and Second persons three composite concepts" at exactly same one position triple completely compound together. And this cause majority people fall in the fog.

"Second person one of two composite concepts 二.A", and, "Second person one of two composite concepts 二.B", both of them are "First person one composite concepts" itself two perfectly half amount body, at same time both of them are "Third and Fourth persons three composite concepts" respective and together two perfectly half amount body also.

"Third and Fourth persons three composite concepts" respective is each other deep part of composition;

"Third and Fourth persons three composite concepts" at approximately same one position triple compound together.

"Third and Fourth persons three composite concepts" all "1" face "First and Second persons three composite concepts" respective, all is composition of equal footing, but they are function all different.

Including all the local natural and all the human being, in "Third and Fourth persons three composite concepts" no any "1" perfect, they all have their own strengths and weaknesses. In the Integrate trend of deep-broad tend to each own and together in have common "Complete-self", all "1" supplementary to each other, and complement each other. In order to sync scientific development, the establishment of modern public morality social system, this paper recommendations mankind to improve life's way, stop use respective strong oppress others weakness that kind of lower animal survival compete mode, respect and follow developed broad and deep of 《The principle of natural law》, civilization-culture of "old, middle-aged, youth and toddler", all the personality together blooming, joint build up a "Pazzo organic democracy complementary Social life". All people live in peace with happiness.

The establishment an inclusive complete national and international network program, who walk in front, who will be the hero that save mankind, Network wise, actions!

God, which is not perfect mankind 's perfect own, blesses humanity!

Not perfect human blesses our own!

Amen!

\section{Author' biography with Photo}

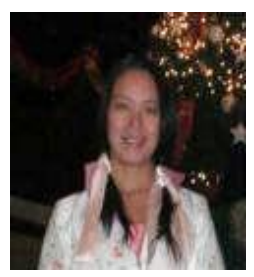

Biography: An ordinary person just like every others, and with many shortcomings and errors need to improve. 